\title{
Consistent assignment of the vibrations of symmetric and asymmetric
}

\section{ortho-disubstituted benzenes}

William D. Tuttle, Adrian M. Gardner, Anna Andrejeva, David Kemp, Jonathan C. A. Wakefield and Timothy G. Wright ${ }^{\mathrm{a}, *}$

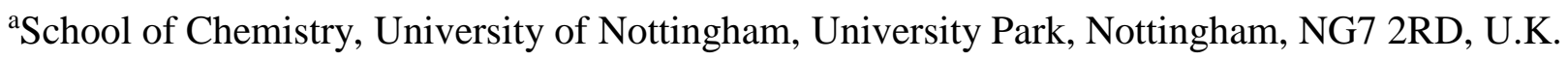

*To whom correspondence should be addressed. Email: Tim.Wright@nottingham.ac.uk

\begin{abstract}
The form of molecular vibrations, and changes in these, give valuable insights into geometric and electronic structure upon electronic excitation or ionization, and within families of molecules. Here, we give a description of the phenyl-ring-localized vibrational modes of the ground $\left(\mathrm{S}_{0}\right)$ electronic states of a wide range of orthodisubstituted benzene molecules including both symmetrically- and asymmetrically-substituted cases. We conclude that the use of the commonly-used Wilson or Varsányi mode labels, which are based on the vibrational motions of benzene itself, is misleading and ambiguous. In addition, we also find the use of the $M_{i}$ labels for monosubstituted benzenes [A. M. Gardner and T. G. Wright. J. Chem. Phys. 135 (2011) 114305], or the recently-suggested labels for para-disubstituted benzenes [A. Andrejeva, A. M. Gardner, W. D. Tuttle, and T. G. Wright, J. Molec. Spectrosc. 321, 28 (2016)] are not appropriate. Instead, we label the modes consistently based upon the Mulliken (Herzberg) method for the modes of ortho-difluorobenzene ( $p$ DFB) under $C_{\mathrm{s}}$ symmetry, since we wish the labelling scheme to cover both symmetrically- and asymmetricallysubstituted molecules. By studying the vibrational wavenumbers from the same force field while varying the mass of the substituent, we are able to identify the corresponding modes across a wide range of molecules and hence provide consistent assignments. We assign the vibrations of the following sets of molecules: the symmetric $o$-dihalobenzenes, $o$-xylene and catechol (o-dihydroxybenzene); and the asymmetric $o$ dihalobenzenes, $o$-halotoluenes, $o$-halophenols and $o$-cresol. In the symmetrically-substituted species, we find a pair of in-phase and out-of-phase carbon-substituent stretches, and this motion persists in asymmetricallysubstituted molecules for heavier substituents. When at least one of the substituents is light, then we find that these evolve into localized carbon-substituent stretches.
\end{abstract}

Keywords: Vibrations; Frequencies; Ground electronic state; ortho-Disubstituted Benzenes 


\section{Introduction}

Vibrations are one of the ways that the spectroscopist gains insight into the geometric and electronic structure of molecules. In particular, vibrational activity during an electronic transition or ionization gives information on the resultant changes in the geometry that occur, which in turn can be related to the details of the orbitals involved. Similarly, changes in vibrational wavenumbers across a family of similar species can give information on changes in electronic structure, once mass effects have been considered. In practice, spectroscopists observe a series of spectral lines, which they then have to assign in terms of the normal modes of the molecule. In a simple picture, the assignments would be in terms of symmetry-allowed fundamentals, overtones and combinations, but further complications can arise from Fermi resonance, and vibronic effects such as Herzberg-Teller coupling.

Comparing spectra between molecules can be difficult as even quite small changes in structure, such as the substitution of a single atom, can lead to significant changes in the appearance of the spectrum. This can arise from a change in symmetry, mass shifts in vibrational wavenumber, and electronic effects. Each of these can change the wavenumbers of vibrations quite significantly. Additionally, in electronic or photoelectron spectra, the electronic structure changes can also induce modifications to the vibrations; furthermore, substituent changes can alter the energy separation of electronic states, and hence the strength of Herzberg-Teller (HT) coupling - this can affect the relative intensities of HT-active vibrations. As a consequence, assigning a vibrationally-resolved spectrum with no further information can be somewhat daunting and almost always comparison to similar molecules is made and, increasingly, to quantum chemical calculations.

In the present work, we focus on substituted benzenes, and note that the comparison of vibrations between such molecules has also been obfuscated by the widespread use of two (or more properly three) main labelling schemes. First, there is comparison of the vibrations of a substituted molecule with those of benzene by using the Wilson mode labels [1]. This does not work because the vibrations of the substituted molecule can be very different to those of the parent benzene: something we have discussed in detail for single-substituted species [2]. A heroic attempt has been made by Varsányi [3] to give Wilson-like labels to an enormous range of substituted benzenes; however, as we point out in [2], different labels were used for the same vibrations when moving between (arbitrarily-defined) "heavy" and "light" substituents. Thus, we dismiss the use of these latter labels, which are labelled "Wilson" or "Varsányi" modes interchangeably throughout the literature. The second set of oft-employed labels are those of Herzberg [4] and Mulliken [5], with "Mulliken labels" now being the more-frequently used term. These are constructed by arranging the vibrations into symmetry classes, taken in a particular order that is given in the second volume [4] of Herzberg's classic texts, and then within each symmetry class, ordering the vibrations in decreasing wavenumber. It immediately becomes clear that comparing between molecules with different symmetries is problematic, as are cases where substituents are 
not single atoms (and so additional vibrations enter the list); moreover, if a substituent group undergoes large amplitude internal motion, such as the internal rotation of a methyl group, then even deciding on what symmetry to use can be an issue.

We have taken a pragmatic approach to this problem, aided by the availability of quantum chemical calculations. Briefly, and as outlined in more detail in Ref. [2] for monosubstituted benzenes, we calculated the force field of benzene using a quantum chemistry code, and then artificially increased the mass of one hydrogen, recalculating the vibrational wavenumbers for each mass within this fixed force field. By plotting the wavenumbers as a function of mass, the variations could be clearly seen. Of note was that these variations had settled down at a mass of around $15 \mathrm{amu}$, and very much smaller changes in wavenumber were observed as the mass was increased further. This turns out to be highly fortuitous, since 14 amu is the mass of an $\mathrm{NH}_{2}$ group, 15 amu that of $\mathrm{CH}_{3}, 17$ amu that of $\mathrm{OH}$ and 19 amu that of $\mathrm{F}$, with other common substituents being of higher mass. We applied this scheme to a wide range of monosubstituted benzenes, focusing on the ringlocalized modes and assuming that substituents were point masses. What was remarkable was that only small perturbations appeared to arise from electronic (mesomeric or inductive) effects, and the variations in wavenumber mostly arose from mass changes. In a series of papers, we have applied this labelling scheme to the vibrationally-resolved electronic spectra of jet-cooled monohalobenzenes [6],[7],[8] and showed that the vibrational activity observed was similar across the series; further application was made to the electronic and high-resolution photoelectron spectra of toluene [9],[10].

It is notable that, in the same way that a single substitution changes the vibrations of benzene significantly, so too does the second substitution on moving from fluorobenzene to $p$-difluorobenzene. As a consequence, different labels are required for the para-disubstituted cases from those in the monosubstituted case (and different to benzene itself), in order to obtain a consistent labelling scheme [11]. This is a little inconvenient, but emphasises that caution is merited in assuming that vibrations given the same Wilson/Varsányi label in different molecules have the same motion. We have applied this approach to symmetrically- and asymmetrically-substituted para-disubstituted benzenes, in examining the induced vibrational activity following electronic excitation and ionization in para-fluorotoluene ( $p \mathrm{FT}$ ) [12], [13], para-xylene (pXyl) [14], [15], and para-chlorofluorobenzene ( $p \mathrm{ClFB}$ ) [16]. With our labelling scheme, we were able to highlight the similarity in vibrational activity in those cases.

Herein, we shall examine the vibrations of ortho-disubstituted benzenes and will conclude that it is not possible to use the Wilson/Varsányi or Mulliken/Herzberg labelling schemes and, further, that it is also not possible to use the monosubstituted or para-disubstituted labels. (In an upcoming paper, we shall demonstrate the same is true for meta-disubstituted benzenes [17].) We shall therefore put forward a separate labelling scheme that covers symmetrically- and asymmetrically-substituted molecules based on the lowest common point group for the family, $C_{s}$ symmetry here, and based on the $o$-difluorobenzene ( $o$ DFB) species. 


\section{Computational Details}

All of the harmonic vibrational frequencies were obtained using B3LYP/aug-cc-pVTZ calculations via the GAUSSIAN 09 software package [18]. For bromine and iodine atoms, the fully relativistic effective core potentials, ECP10MDF and ECP28MDF respectively, were used with corresponding aug-cc-pVTZ-PP valence basis sets. All of the calculated harmonic vibrational wavenumbers were scaled by the usual factor of 0.97 as an approximate method of obtaining anharmonic wavenumber values. This level of calculation has been shown to be very reliable for the $S_{0}$ vibrational wavenumbers of a range of substituted benzenes [2], [11]. In the following, we shall calculate vibrational wavenumbers for the actual molecule, but also vibrational wavenumbers that are calculated using one molecule's force field, but then artificially changing the mass of one or two atoms to match those of substituents (atomic or otherwise). In this way, we can map out changes in the vibrational wavenumbers that occur solely from the mass effect and any deviations from these. In addition, we shall calculate generalized Duschinsky matrices using FC-LabII [19] to illustrate the mixed nature of the vibrations of one molecule when expressed as those of another. In each case we show results for the lowest energy conformer we found at the specified level of theory, although in the case of $o$-xylene the search was not exhaustive owing to convergence issues for some orientations. In any case, experience has shown that we do not expect the conformation to affect the phenyl-localized modes to any great extent for these molecules, and it is certainly the case that much higher levels of theory would be required to obtain quantitative energy differences between some of these conformers.

\section{Labelling the $\mathrm{S}_{0}$ vibrational modes of $o \mathrm{DFB}$}

We have covered much of the background to our methodology in refs. [2] and [11]; hence, we present the results succinctly here.

As a first step, we compare the vibrations of $o \mathrm{DFB}$ with those of benzene. There is an immediate problem, since a choice of axis system must be made. The $C_{2}$ axis in $o$ DFB bisects the $\mathrm{C}-\mathrm{C}$ bond that has the two fluorine atoms attached to it, and so is the $z$ axis according to convention; in contrast, the $C_{6}$ axis in benzene is perpendicular to the molecular plane, and by convention is the $z$ axis. Hence, in moving from benzene to an $o$-disubstituted benzene molecule, then the molecule moves from lying in the $x y$ plane for benzene to the $y z$ plane for an $o$-disubstituted benzene molecule. However, these axis systems are just conventions, and so for ease of comparison, we compare the vibrations of benzene and $o$ DFB with both molecules lying in the $y z$ plane; further, to compare motions the atoms in the molecules need to be aligned, and we align the molecules in an "obvious" relative orientation as shown in Figure 1.

Figure 1 represents the vibrations of $o$ DFB in terms of those of benzene using a Duschinsky matrix approach, where one set of vibrations are expressed as linear combinations of another set. As noted in the caption, the shading indicates how well the vibration of one molecule represents that of the other, with black meaning the 
vibrations are identical, white meaning there is essentially no resemblance, and the depth of shade of grey representing the degree of similarity. As may be seen from this figure, the modes fall nicely into $C_{2 v}$ symmetry blocks, but there is mixing between the modes: that is, the Wilson modes of benzene are not a good representation of the normal modes of $o \mathrm{DFB}$, and this is true across the four $C_{2 v}$ symmetry classes. It is interesting to note that while the $6 \mathrm{a} / \mathrm{b}, 9 \mathrm{a} / \mathrm{b}$ etc. degenerate vibrations of benzene separate into $a_{1} / b_{2}$ or $a_{2} / b_{1}$ pairs, matching the corresponding $a / b$ label in each case is not true, owing to the location of the $z$ axis; for example, the $18 \mathrm{~b}$ mode is of $a_{1}$ symmetry and the $18 a$ mode is of $b_{2}$ symmetry, while the reverse separation occurs for the $9 a / b$ vibrations.

In setting up the Duschinsky matrix, we have noted that the molecules need to be aligned with atoms in corresponding positions, but some caution is required in doing this. So, in the present case, if we rotate the benzene molecule's axis system relative to that of $o$ DFB by $60^{\circ}$, but keep the same atomic motions associated with the same atoms, then we still have an alignment of the atoms, but find that there is mixing between the $a_{1}$ and $b_{2}$ modes, and also between the $a_{2}$ and $b_{1}$ modes, and so the relative orientation shown in Figure 1 is the more natural one. (The reader can visualize this by looking at the motion of the $v_{9 a}$ mode shown to the right in Figure 1 and seeing that it is a close match to that of ${ }^{\circ} D_{5}-$ see below for a description of this labelling - but if the benzene molecule is rotated by $60^{\circ}$, there is still alignment of the atoms, but the motions no longer correspond.) In summary, our conclusion is that the association of the benzene Wilson modes with those in an $o$-disubstituted benzene molecule is even more problematic than in the monosubstituted [2] or paradisubstituted cases [11].

In Table 1 we present the data used to make up Figure 1 in a different way, with the notation explained in the footnotes. As may be seen from both Figure 1 and Table 1, the $o$ DFB normal modes are significant mixtures of the benzene ones and in many cases it is not possible to associate a unique Wilson label with a particular $o$ DFB normal mode.

We now compare the $o$ DFB normal modes with those of monofluorobenzene (FBz) (the $M_{i}$ modes of Ref. [2]) using a similar approach. As noted above, to construct the Duschinsky matrix, the atoms must be in the same orientations, i.e. the molecules need to be aligned; however, there is no unique way of doing this. We show what we feel is the most obvious way of comparing the modes, by aligning the $\mathrm{C}-\mathrm{F}$ bond in $\mathrm{FBz}$ with one such bond in $o$ DFB. The resulting Duschinsky matrix is shown in Figure 2, and the breakdowns of the $o$ DFB modes in terms of the FBz ones are given in Table 1. As may be seen from Figure 2, there is not a good match between the FBz and $o$ DFB modes, with the mixing being considerable, and only a small number of vibrations of $o$ DFB are largely identifiable with a single $M_{i}$ mode, with the others being significant mixtures of modes.

We now consider how the vibrations of benzene evolve into those of $o$ DFB as we simultaneously (and artificially) change the masses of two adjacent hydrogens from $1 \mathrm{amu}$ to $19 \mathrm{amu}$ - this allows the mass-only effects on the vibrational wavenumbers to be examined. We have done this by aligning the axis systems of 
benzene and $o$ DFB as we did above, which separates the vibrations into $C_{2 v}$ symmetry classes. In Figure 3 we show the full-range plots for the $a_{1}$ and $b_{2}$ symmetry vibrations, while in Figure 4 we show the plots for all four $C_{2 v}$ symmetry classes, but omitting the high-wavenumber vibrations for the $a_{1}$ and $b_{2}$ symmetry classes. We first consider the evolution of the modes from benzene to $o \mathrm{DFB}$, which correspond to the left-hand sides of each quadrant. There are significant changes in the wavenumbers of many of the vibrations as the mass changes, and interestingly a number of the curves look like they undergo "avoided crossings". We interpret these as the normal modes changing and becoming significant admixtures of the original normal modes, within the same symmetry class. Notably, once the artificial masses reach $\sim 10 \mathrm{amu}$ the curves become more settled, which we have taken to imply that the form of the normal modes are now largely stable and this persists to higher mass. In the case of the p-disubstituted benzenes in Ref. [11], we gave detailed commentaries on the evolution of the benzene modes as the mass increased and the interested reader can look at Ref. [11] and then apply the same ideas while looking at the left-hand side of the present Figures 3 and 4, while comparing to the breakdowns given in the present Table 1. Again, it is clear that some modes are very mass sensitive, with the motion altering significantly at low masses, causing the changing wavenumber. The changes in motion lead to the evolving modes being represented by linear combinations of the original Bz modes. The summary of this analysis is similar to that in the previous paragraph: it is not possible to use the Wilson mode labels for the vibrations of $o$-disubstituted benzenes - a corresponding conclusion also reached in the case of $p$ disubstituted benzenes [11].

We now explain why we do not simply use the ${ }^{p} D_{i}$ labels that we deduced previously for the para-disubstituted benzenes [11]. On the right-hand sides of Figures 3 and 4, again we have taken the force field for benzene, but now change the mass of two opposite hydrogen atoms from 1 to 19 amu simultaneously, as indicated. For the purposes of this comparison, we have aligned the molecules as shown. This maintains each vibration in the same $C_{2 v}$ symmetry class as we change the masses, and so allows the correlation of $p$ DFB vibrations on the right-hand side with $o$ DFB vibrations on the left-hand side, via the vibrations of benzene in the centre. Note that this is not the same $C_{2 v}$ axis system employed in Ref. [11], and is used here simply to allow a more direct comparison of the ortho and para vibrational motions; note that this also allows the atoms and the axis systems to remain aligned. However, the numbering of the $p$ DFB modes given on the right-hand side of the diagram does correspond to that given in Ref. [11]. Use of the same axis system also allows us to deal with the fact that with the original axis system used for $p$ DFB [11] there are $3 a_{2}+6 b_{2}$ out of plane modes, while in the system used here for $p \mathrm{DFB}$ and $o \mathrm{DFB}$ there are $5 a_{2}+4 b_{1}$ such modes. In addition, although there are the same number of each of the $a_{1}$ and $b_{2}$ in-plane modes in the two axis systems, and the same vibrations belong to one of these two classes, some vibrations change their symmetries between the two axis systems. Further, when considered in its full $D_{2 h}$ point group, the $p$ DFB vibrations have $u$ and $g$ symmetry. To help the reader identify the various vibrations in the diagram, we have used different colours for each of the $a$ and $b$ symmetry vibrations across the two $a_{1}, b_{2}$ and $a_{2}, b_{1}$ pairs of classes, as indicated; we also used dashed lines to imply $u$ 
symmetry and solid lines for $g$ symmetry. As may be seen from Figure 4, this means that lines where the colour or line type is different can cross (as they have different $D_{2 h}$ symmetries), while if these are both the same, then the vibrations mix and an avoided crossing is observed. (Note that in Figure 1 of Ref. [11] the first point was not correctly indicated, so that the ${ }^{p} D_{19}$ and ${ }^{p} D_{18}$ modes can cross, for example.) So, looking at the $a_{1}$ vibrations in the top right panel, we see that the ${ }^{p} D_{3}$ and ${ }^{p} D_{5}$ vibrations interact and undergo an avoided crossing, while the ${ }^{p} D_{11}$ and ${ }^{p} D_{30}$ vibrations can cross. On the left-hand side, it is the case that no curve can cross (as they all have the same symmetry in the full point group of the molecule), and so, for example, we see a clear avoided crossing between the ${ }^{o} D_{18}$ and ${ }^{o} D_{21}$ vibrations. Further consideration of these curves, together with the breakdowns provided in Table 1, give insight into the interactions/mixings that are occurring between different vibrations; it is particularly interesting that there are some contributions from the highest $a_{1}$ modes into the lowest ones. Similar comments also apply to the other classes.

It is clear that large mass-dependent changes are occurring early on as we move away from benzene in the centre of the figure. It is true again, however, that the changes largely settle down, from about 15 amu onwards. Evidently, if we start on the far right-hand side of the diagrams in Figures 3 and 4 and trace a mode from $p$ DFB through benzene to $o$ DFB, we will have encountered various avoided crossings and, therefore, mixings of the modes. If we compare the motions on the far left- and far right-hand sides via a Duschinsky matrix approach, as shown in Figure 5, we can see that there are very few modes that have the same motions for both $o \mathrm{DFB}$ and $p \mathrm{DFB}$. For these reasons, we have had to accept that the labelling scheme for the ortho-disubstituted benzenes will need to be different to that of the para-disubstituted molecules. Indeed, the significant changes in the modes implied by Figure 5 provides a general word of caution in trying to compare vibrations between different isomers of substituted benzenes. Note that in certain special cases, for example replacing the ortho hydrogen in fluorobenzene by a deuterium atom, there will still be significant mixing of modes and so more caution would need to be exerted; we discussed this point in depth in the case of the monosubstituted species [2], looking at monodeuterated benzene.

Having concluded that we need to employ a new labelling scheme for ortho-disubstituted benzenes, we now look at whether employing the modes of $o \mathrm{DFB}$ as the basis for this is sound. In Figure 6, mass-correlation diagrams are presented that employ the force-field of $o$ DFB whilst simultaneously changing the masses of both fluorine atoms artificially to that of each of the naturally-occurring isotopes of the halogens with the highest abundance. It may be seen there that, although there is a trend for the wavenumber to lower with increasing mass as expected, there is little indication of significant mixing between the modes and this is confirmed by Duschinsky matrices (not shown). Additionally, in Figure 7, we show a mass-correlation diagram again using the force field of $o \mathrm{DFB}$ and changing the mass of only the ortho fluorine from mass 19 to mass 127, and again little mixing is seen, which is again confirmed by Duschinsky matrices (not shown). As a consequence, and in line with similar conclusions outlined in our earlier papers [2, 11], we take the normal modes of $o$ DFB as the basis for the labels of ortho disubstituted molecules, with the label numbers 
being given by the standard Mulliken scheme [5]. However, because we wish the labels to be applicable across both symmetrically- and asymmetrically-substituted molecules, we construct these labels in the $C_{\text {s }}$ point group, and denote them ${ }^{o} D_{i}$, with the presuperscript to be included only when required to avoid ambiguity considering different isomers, and $i$ running from 1 (for the highest wavenumber $a^{\prime}$ vibration) to 30 (for the lowest $a^{\prime \prime}$ vibration). Note that our labels are restricted to the phenyl-ring localized modes, and so any substituents are effectively treated as point masses when determining the ${ }^{o} D_{i}$ labels, with substituent-localized vibrations being treated separately, as required. This means that even in cases where the substituents lower the overall point group symmetry to $C_{1}$, the proposed scheme is still valid.

In summary, we propose the use of ${ }^{o} D_{i}$ labels for the normal modes of the phenyl-ring-localized vibrations of ortho-disubstituted benzenes. The normal mode diagrams are indicated in Figure 8 and it is relatively straightforward to assign a label to a vibration with a particular wavenumber, based either on expected mass trends, or by calculating and visualizing the motion with widely-available quantum chemical software. As examples of this, in Figure 9 we show the calculated motions of five $o \mathrm{DFB}, o \mathrm{ClFB}$ and $o \mathrm{DCB} a^{\prime}$ modes. The ${ }^{o} D_{11}$ and ${ }^{o} D_{13}$ modes look very similar across the series, with just small variations in motion in line with keeping the centre of mass fixed with the changing halogen masses. The ${ }^{o} D_{10},{ }^{o} D_{12}$ and ${ }^{o} D_{14}$ modes have some similarities, but can be identified by noting the following. ${ }^{o} D_{10}$ is either a symmetric, in-phase, stretch of the two substituents, or is the higher of the localized C-X and C-Y stretches, where $\mathrm{X}$ and $\mathrm{Y}$ are substituents. ${ }^{\circ} D_{12}$ is either an asymmetric, out-of-phase, stretch of the two substituents, or is the lower of the localized C-X and $\mathrm{C}-\mathrm{Y}$ stretches, where $\mathrm{X}$ and $\mathrm{Y}$ are substituents. ${ }^{o} D_{14}$ is identifiable as having out-of-phase bending of the pairs of $\mathrm{C}-\mathrm{H}$ bonds on opposite sides of the ring.

\section{Assigning the vibrations}

We shall now consider four families of ortho-disubstituted benzenes, assign ${ }^{o} D_{i}$ labels and discuss previous assignments where appropriate, these are: symmetric disubstituted benzenes involving halogens, $\mathrm{OH}$ or $\mathrm{CH}_{3}$; and the asymmetric dihalobenzenes, halotoluenes, halophenols and cresol. We have found it straightforward to identify the ${ }^{o} D_{i}$ label from the calculated motion in all of these cases, as discussed below.

\subsection{Symmetric disubstituted benzenes}

A summary of the available experimental and the present calculated vibrational wavenumbers are presented in Table 2. The thirty vibrational wavenumbers of the ground electronic state $\left(\tilde{X}^{1} A_{1}\right)$ of the $C_{2 v}$ molecule, $o$ DFB have been summarized by Varsányi [3], with Wilson-like labels given to these; the readily-available values were cited as being taken from two papers by Green and coworkers [20],[21] and one by Steele [22], with cited work in Green [20] also including Raman studies from Refs. [23], [24] and [25]. Most of those values are from infrared or Raman studies of the liquid, although the infrared ones for $p$ DIB are from studies of a solution. We report a summary of the values in Table 2, favouring the infrared values over the Raman 
values where both have been given. In a few cases, to give vibrations with similar motions the same ${ }^{o} D_{i}$ label, a couple of switches in wavenumber ordering from that in $o$ DFB was required - in these cases we have also reordered the experimental vibrational wavenumbers to match. The values for $o$ DFB were slightly modified by Swinn and Kable [26] in their laser-induced fluorescence/dispersed fluorescence study of jet-cooled gaseous molecules, who also gave Mulliken labels together with Varsányi ones; interestingly, from band positions and assignments given in that work [26], slightly different $S_{0}$ values can be derived, which we would expect to be more comparable with the calculated values. As a consequence, where available, these values have been preferred in Table 2. In keeping with the construction of the ${ }^{o} D_{i}$ labels, we have separated the vibrations into $a^{\prime}$ and $a^{\prime \prime}$ symmetry, but have also indicated both the $C_{2 v}$ Mulliken numbering (as used in Ref. [26], for example), as well as the $\mathrm{C}_{2 v}$ symmetry, in Table 2. In the following, the dihalogens are abbreviated in an obvious way, as we have done for $o \mathrm{DFB}$, and $o \mathrm{Xyl}=$ ortho-xylene (xylene $=$ dimethylbenzene). The corresponding vibrational wavenumbers for $o \mathrm{DClB}, o \mathrm{DBrB}, o \mathrm{DIB}$ and $o \mathrm{Xyl}$ are all from Green [20]. For catechol (also known as pyrocatechol, $o$-dihydrobenzene or benzene-1,2-diol), there have been a number of studies [23, 27, 28, 29, 30, 31] and we have favoured the gas-phase values of Wilson [29] and then the morerecent values on the liquid [31] over the earlier values summarized in [27], where available. We have deduced the assignments from the given symmetry and comments given in these papers, with a few discussion points given below. (Note that the conformers used for catechol and $o \mathrm{Xyl}$ are given in a footnote to Table 2.)

In Table 2, we give our calculated wavenumbers, and it may be seen that there is generally very good agreement with the experimental values across the board, with there being only a small number of missing experimental values. In most cases, it is straightforward to track the vibrational modes across this family of molecules, particularly as many of the original experimental assignments were able to deduce the $C_{2 v}$ symmetry class of a vibration from a band profile and/or comparing infrared and Raman activity. There are several points to highlight. For the heavier dihalobenzenes we find that we need to switch the ordering of the $D_{10}$ and $D_{13}$ vibrational wavenumbers, and also the $D_{12}$ and $D_{14}$ vibrations. For $p$ DIB we also see that the calculated value of $308 \mathrm{~cm}^{-1}$ for $D_{19}$ is not in good agreement with the experimental value of $206 \mathrm{~cm}^{-1}$. In fact, the latter value was noted in the text has having an uncertain assignment, and it is not clear why it was associated with the given fundamental. Given the coincidence of the calculated values for $D_{19}$ and $D_{18}$, it is possible that the experimental values for these were overlapped. It is possible that the two values noted in Ref. [20] of $200 \mathrm{~cm}^{-1}$ and $206 \mathrm{~cm}^{-1}$ could actually be assigned to the $D_{21}$ first overtone and the $D_{20}$ respectively, and we currently hypothesise this. We thus conclude that the assignment of the $206 \mathrm{~cm}^{-1}$ band in $p$ DIB to $D_{19}$ is erroneous and reassign this to $D_{20}$; we then hypothesise that the $D_{18}$ and $D_{19}$ bands are coincident. Figure 6 shows a comparison of the experimental values to the ones calculated from the $o$ DFB force-field and using artificial isotopes.

For catechol, there have been a number of infrared and Raman studies on the liquid, and one also on the vapour. We have preferred the values from the vapour studies [29], and then those from the recent work of 
Koh et al. [31]. We note that the value for $D_{9}$ from the vapour study would be $1364 \mathrm{~cm}^{-1}$, but a fundamental in that work of $1324 \mathrm{~cm}^{-1}$ (assigned to an OH-localized vibration therein) was preferred by Koh et al. [31] and this value appears to be in better agreement with the calculated value herein. We note that assignments of three phenyl-ring localized vibrations at $1275 \mathrm{~cm}^{-1}, 1195 \mathrm{~cm}^{-1}$ and $1035 \mathrm{~cm}^{-1}$ in Ref. [29] seem to be erroneous based on the present calculated values. Indeed, some assignments have been modified slightly in Ref. [28] and again in Ref. [31] with some uncertainty implied as to which vibrations are OH-localized and which are phenyl-ring localized. Lastly, the value of $501 \mathrm{~cm}^{-1}$ reported for one of the in-plane vibrations in Ref. [27] also seems to be erroneous based on later studies and our calculated values.

It is interesting to note that the $D_{10}$ and $D_{12}$ vibrations may be well described as in-phase and out-of-phase $\mathrm{C}-\mathrm{X}$ stretches, where $\mathrm{X}$ is a substituent, a similar situation was observed in the para-disubstituted species and in Ref. [11] we noted that these modes evolved into largely localized C-X and C-Y stretches in the asymmetric species, where $\mathrm{X}$ and $\mathrm{Y}$ are different substituents.

\subsection{Asymmetric dihalobenzenes}

A summary of the available experimental and the present calculated vibrational wavenumbers are presented in Table 3. The experimental values for the asymmetric $o$-dihalobenzenes have been taken from Ref. [20] which are mostly infrared spectroscopic data for the liquid, but with some Raman values taken from Refs. [24], [32] and [33] (also summarized in Ref. [20]). To obtain consistency, we have given vibrations with similar motions the same $D_{i}$ label, which sometimes leads to the vibrations being in a different wavenumber order to those in $o \mathrm{DFB}$ - in these cases we have also reordered the experimental vibrational wavenumbers to match. As may be seen, there are only a small number of missing experimental values, and in general there is good agreement across the whole series. With the given assignments, it is again possible to track the same vibration across the series of molecules. We find that the order of vibration $D_{10}-D_{15}$ has changed from that in $o$ DFB and so these are not in wavenumber order; however, the motions of modes given the same $D_{i}$ number are a good match across the series, meaning the assignment is sound. We note that the experimental value of the $D_{18}$ band for $o$ CIIB of $366 \mathrm{~cm}^{-1}$ [20] is in poor agreement with the calculated value of $425 \mathrm{~cm}^{-1}$; in fact the $366 \mathrm{~cm}^{-1}$ value could be associated with the $20^{1}+21^{1}$ combination band, and so we think this value for $D_{18}$ in Ref. [20] is incorrect.

As noted in the previous sub-section, from our work on the para-disubstituted species, we would expect the $D_{10}$ and $D_{12}$ vibrations to evolve into localized $\mathrm{C}-\mathrm{X}$ and $\mathrm{C}-\mathrm{Y}$ stretches and in a number of cases, this seems to be the case and so we label these vibrations with this character in Table 3. In such cases, and in a similar way to what we did in Ref. [11], we have given the label $D_{10}$ consistently to the highest wavenumber C-X stretch and the label $D_{12}$ to the lowest wavenumber C-Y stretch. We note that there are some other vibrations with various small amounts of $\mathrm{C}-\mathrm{X}$ and $\mathrm{C}-\mathrm{Y}$ stretch character, and so this localization of motion is not complete, but it is a good approximation in some cases, and we find that the $D_{10}$ vibrational wavenumbers of 
the ortho-disubstituted benzenes are generally similar to the $D_{5}$ vibrational wavenumbers for the corresponding para ones [11]. When the lightest substituent is $\mathrm{Cl}$ or heavier, this localization does not occur - we think this is related to the distorted motion - and so in such cases we retain the symmetric and asymmetric labelling in Table 3.

\section{3 o-halotoluenes}

A summary of the available experimental and the present calculated vibrational wavenumbers for the phenylring-localized vibration of the four commonly-occurring $o$-halotoluenes are presented in Table 4. (The conformers employed are shown in a footnote to this table.) Again, these values come from the work of Green [20] and are mostly infrared spectroscopic values obtained on the liquid, with a few being from Raman studies [25]. Again, we have given vibrations with similar motions the same $D_{i}$ label, which sometimes leads to the vibrations being in a different wavenumber order to those in $o \mathrm{DFB}$ - in these cases we have also reordered the experimental vibrational wavenumbers to match. Again, there are several missing experimental values, but there is good agreement across the whole series and so the given assignments allow the same vibration to be tracked across the series of molecules. The reader will recall that the ${ }^{o} D_{i}$ labels only cover the phenyl-ring localized motions, and so do not include the vibrations largely localized to the methyl group, which form a separate set. Experience from our previous work $[2,11]$ and herein show that coupling between the methyllocalized and ring-localized sets of vibrations seems to be small, but will clearly be present and so may need to be taken account of in higher precision measurements. However, for the infrared and Raman studies referred to here, and our application to vibrationally-resolved electronic excitation (and dispersed fluorescence) spectra mentioned in the above text, the localized sets appear to be sufficient.

Here we find a localization of the $\mathrm{C}-\mathrm{X}$ and $\mathrm{C}-\mathrm{CH}_{3}$ stretch motions across the series, and in a similar manner to the convention we adopted in Ref. [11], we use $D_{10}$ always to label the carbon-halogen stretch and then $D_{12}$ to label the $\mathrm{C}-\mathrm{CH}_{3}$ stretch (labelled $\mathrm{C}-\mathrm{Me}$ ) in Table 4.

\section{3 o-halophenols and o-cresol}

A summary of the available experimental and the present calculated vibrational wavenumbers for the phenylring localized vibrations of the $o$-halophenols and o-cresol are presented in Table 5. (The conformers employed are shown in a footnote to this table.) These are taken from Green [34] and are mainly infrared spectroscopic studies of liquids, but with some values taken from a Raman study [25], but cited in Ref. [34]. We have given vibrations with similar motions the same $D_{i}$ label, which sometimes leads to the vibrations being in a different wavenumber order to those in $o \mathrm{DFB}$ - in these cases we have also reordered the experimental vibrational wavenumbers to match. Once again, the agreement between the calculated and experimental values is generally very good and trends in the vibrations can be followed across the series. Again, we note that vibrations localized to the hydroxyl groups, or methyl group in the case of $o$-cresol, are 
not part of the ${ }^{o} D_{i}$ vibrational set. It is interesting to note that there is no conclusive evidence for perturbation of any of the out-of-plane phenyl-ring localized vibrations, even when the substituents are moving in opposite directions relative to the ring (see mode diagrams in Figure 8 and values in Table 5). This is a little surprising as it may be expected that there would be some hydrogen-bonding-type interaction between the hydroxyl group and the halogen. Further evidence for the absence of any significant interaction comes from the similarity of the $o$-cresol vibrational wavenumbers and those of $o \mathrm{FPhOH}$ where the masses are quite similar.

We find localization of the $\mathrm{C}-\mathrm{OH}$ and $\mathrm{C}-\mathrm{X}$ stretch motions in most cases, and in a similar manner to the convention we adopted in Ref. [11], we use $D_{10}$ to label the $\mathrm{C}-\mathrm{OH}$ stretch and $D_{12}$ to label the carbon-halogen, or carbon-methyl (labelled C-Me) stretch in Table 5. The exception is for $o \mathrm{FPhOH}$, where this localization does not occur cleanly, and so we retain the symmetric/asymmetric labels for $D_{10}$ and $D_{12}$ in this case.

\section{Conclusions}

In the present work, we have shown that it is possible to label the phenyl ring-localized vibrations of a range of ortho-disubstituted benzenes consistently, such that vibrations with the same atomic motion have the same label. In doing this we have seen that the ordering of the vibrations changes between species - this means that application of the usual Mulliken (Herzberg) labelling scheme for each species would give different labels to the same vibrations. We have shown how the ortho motions are related to those of the other species, and shown that these are often heavily mixed versions of each other. This leads to the conclusion that the vibrational motions of the ortho-disubstituted benzenes are significantly different to those of benzene, monosubstituted benzenes or para-disubstituted species. This means both that different labelling schemes are required and, further, that some caution is required when drawing conclusions on vibration-specific trends across different isomers. It is worth noting that the centre-of-mass in benzene, monosubstituted benzenes and para-disubstituted benzenes stays on the $z$-axis (at least for the most part) whether the substitution is symmetric or not, while for asymmetric ortho-disubstituted species, the centre of mass migrates towards the heavier substituent, causing significant distortions of the motions of the other atoms, to compensate. Despite this, it was still possible to trace the motions as a function of mass for a fixed force field, but also to be able to identify to which of the ${ }^{o} D_{i}$ motions (given in Figure 8) a calculated vibration corresponded.

As in the para-disubstituted benzenes [11], in a number of cases we were able to discern the localization of the in-phase and out-of-phase motions of the carbon-substituent bond stretching motions from the symmetrically- to the asymmetrically-substituted species. We have thus labelled these vibrations consistently, in a similar way to that used in Ref. [11].

We anticipate that the use of this labelling scheme for ortho-disubstituted benzenes will reveal trends in vibration-specific behaviour between species more clearly, as we have found when examining the 
vibrationally-resolved electronic and photoelectron spectra of both monosubstituted and para-disubstituted benzenes.

\section{Acknowledgements}

We are grateful to the EPSRC for funding via grant EP/L021366/1. The EPSRC and the University of Nottingham are thanked for studentships to A.A. and W.D.T. We are grateful to the NSCCS for the provision of computer time under the auspices of the EPSRC, and to the High Performance Computer resource at the University of Nottingham. 


\section{Figure Captions}

Figure 1: Generalized Duschinsky matrix showing how the vibrational modes of $o$ DFB can be expressed in terms of the benzene ones - clearly significant mixing of the benzene modes occurs - see text for further discussion. Black shading indicates a normalized coefficient value of 1.00 , while white indicates a value of 0.00 , with grey shading indicating intermediate values, see text for details.

Figure 2: Generalized Duschinsky matrix showing how the vibrational modes of $o$ DFB can be expressed in terms of the FBz ones - clearly significant mixing of the fluorobenzene modes occurs - see text for further discussion. Black shading indicates a normalized coefficient value of 1.00 , while white indicates a value of 0.00 , with grey shading indicating intermediate values, see text for details. Note that although the vibrations can each be labelled with a $C_{2 v}$ symmetry class, these would be different for $\mathrm{FBz}$ and $o \mathrm{DFB}$, as the $C_{2}$ axis and atoms cannot be simultaneously aligned in the two molecules.

Figure 3: Mass-correlated vibrational wavenumbers showing how the vibrations of benzene (centre) evolve into those of $p \mathrm{DFB}$ (right) and $o \mathrm{DFB}$ (left) for the $a_{1}$ and $b_{2}$ vibrations, according to the indicated axis system. The force field of benzene is employed and the masses of the indicated hydrogen atoms are artificially varied from 1 to 19 amu in each case: ${ }^{19} \mathrm{H}$ in the figure indicates that the mass of the indicated hydrogen atom has been artificially changed to 19 amu. The $o$ DFB labels have been included on the left, while on the right hand side the numbers refer to the ${ }^{p} D_{i}$ modes as in Ref. [11]. An expanded region of both of these plots is shown as part of Figure 4, which also gives the Wilson modes in the centre. Note that we have used the same colour scheme and line type as employed in Figure 4.

Figure 4: Mass-correlated vibrational wavenumbers showing how the vibrations of benzene (centre) evolve into those of $p \mathrm{DFB}$ (right) and $o \mathrm{DFB}$ (left) for the vibrations separated into the four $C_{2 v}$ symmetry classes, according to the indicated axis system. The force field of benzene is employed and the masses of the indicated hydrogen atoms are artificially varied from 1 to 19 amu in each case: ${ }^{19} \mathrm{H}$ in the figure indicates that the mass of the indicated hydrogen atom has been artificially changed to $19 \mathrm{amu}$. The $o$ DFB labels have been included on the left, on the right hand side the numbers refer to the ${ }^{p} D_{i}$ modes from Ref. [11], while the Wilson modes are given in the centre. To aid the reader further, we have also indicated the appropriately colour-coded $D_{2 h}$ symmetry classes on the right-hand side of each panel. To allow comparison with Ref. [11], the pink/red lines indicate $a_{1}$ or $a_{2}$ symmetry vibrations, and blue/green lines indicate $b_{1}$ or $b_{2}$ vibrations, respectively - in each case now referring to the axis system used in that work. Further, vibrations on the right-hand side belong to the $D_{2 h}$ point group and so have $g / u$ symmetry - this is indicated by solid/dashed lines respectively. See text for further discussion.

Figure 5: Generalized Duschinsky matrix showing how the vibrational modes of $o$ DFB can be expressed in terms of the $p$ DFB ones, with the molecules aligned as indicated - clearly significant mixing of the modes occurs - see text for further discussion. Black shading indicates a normalized coefficient value of 1.00 , while white indicates a value of 0.00 , with grey shading indicating intermediate values, see text for details. The alignment given allows separation of the modes for both molecules into $C_{2 v}$ symmetry blocks, but we have also indicated the $C_{s}$ symmetry partition that is appropriate for asymmetrically-substituted ortho-disubstituted benzenes, and as used for the construction of the ${ }^{o} D_{i}$ labels. 
Figure 6: Mass-correlated vibrational wavenumbers showing how the vibrations of $o$ DFB (left-hand side) evolve as a function of mass in symmetric dihalo species; the highest wavenumber vibrations have been omitted (refer to Table 2). The vibrations have been separated into the two $C_{s}$ symmetry classes, and in each class we have indicated the $a$ or $b$ character with colour, as indicated. The force field of $o \mathrm{DFB}$ is employed and the masses of both fluorine atoms are artificially varied from 19 to $127 \mathrm{amu}$ simultaneously in each case, to match the mass of the most abundant, naturallyoccurring isotope of the halogens. The numbering of the ${ }^{o} D_{i}$ labels is included on the left. Note that crossings between vibrations of different symmetry is allowed.

Figure 7: Mass-correlated vibrational wavenumbers showing how the vibrations of $o$ DFB (left-hand side) evolve as a function of mass in asymmetric dihalo species; the highest wavenumber vibrations have been omitted (refer to Table 3). The vibrations have been separated into the two $C_{s}$ symmetry classes. The force field of $o$ DFB is employed and the mass of the ortho fluorine atom is artificially varied from 19 to $127 \mathrm{amu}$ in each case, to match the mass of the most abundant, naturally-occurring isotope of the halogens. The numbering of the ${ }^{o} D_{i}$ labels is included on the left. (Note that the theoretical lines do not cross for $D_{10}$ and $D_{11}$, as required; however, the experimental data indicate that there is a switch in the ordering of these two vibrations between $p \mathrm{DFB}$ and the cases where one fluorine atom is replaced by a heavier halogen - this effect is very small, and must be a result of subtle changes in the force field. The actual assignments of the vibrations, however, are based on the atomic motion.)

Figure 8: Calculated vibrational modes for $o$ DFB (B3LYP/aug-cc-pVTZ), labelled using the ${ }^{o} D_{i}$ notation in the present work. See text for details.

Figure 9: A selection of calculated vibrational modes for $o \mathrm{DFB}, o \mathrm{ClFB}$ and $o \mathrm{DClB}$ (B3LYP/aug-cc-pVTZ), labelled using the ${ }^{o} D_{i}$ notation in the present work. (Green circle represents $\mathrm{F}$ and a red circle represent $\mathrm{Cl} ; \mathrm{C}$ and $\mathrm{H}$ are both open circles.) Note that the $D_{10}$ and $D_{12}$ modes are largely the in-phase and out-of-phase C-F (or C-Cl) stretches for $o$ DFB $(o \mathrm{DClB})$, while in $o \mathrm{ClFB}$ these modes have evolved in being largely-localized $\mathrm{C}-\mathrm{F}$ and $\mathrm{C}-\mathrm{Cl}$ stretches, respectively. See text for details and discussion. 
Table 1. Labelling schemes for the $S_{0}$ vibrations of $o$ DFB

\begin{tabular}{|c|c|c|c|c|}
\hline Mode $^{a}$ & $\begin{array}{c}\text { Mulliken } \\
C_{2 v}{ }^{\mathrm{b}}\end{array}$ & $o$ DFB - Varsányic & Mixed $(B z)^{c, d}$ & $\operatorname{Mixed}(\mathrm{FBz})^{\mathrm{e}}$ \\
\hline \multicolumn{5}{|c|}{$a^{\prime}$} \\
\hline${ }^{o} D_{1}\left(\mathrm{a}_{1}\right)$ & 1 & 2 & $\mathbf{2}, 20 \mathrm{~b}(7 \mathrm{a})$ & $\boldsymbol{M}_{1}, M_{2}\left(M_{21}\right)$ \\
\hline${ }^{o} D_{2}\left(\mathrm{~b}_{2}\right)$ & 21 & $7 b$ & 20a(13,7b) & $M_{21}, M_{2}, M_{22}$ \\
\hline${ }^{o} D_{3}\left(\mathrm{a}_{1}\right)$ & 2 & $20 \mathrm{~b}$ & $\mathbf{7 a}, 20 \mathrm{~b}$ & $\mathbf{M}_{22}, M_{2}$ \\
\hline${ }^{o} D_{4}\left(\mathrm{~b}_{2}\right)$ & 22 & $20 \mathrm{a}$ & $13,7 \mathrm{~b}$ & $\mathbf{M}_{3}\left(M_{22}\right)$ \\
\hline${ }^{o} D_{5}\left(\mathrm{a}_{1}\right)$ & 3 & $8 \mathrm{a}$ & $9 \mathbf{a}$ & $\mathbf{M}_{23}, M_{4}$ \\
\hline${ }^{o} D_{6}\left(\mathrm{~b}_{2}\right)$ & 23 & $8 \mathrm{~b}$ & $9 b$ & $M_{4}, M_{23}$ \\
\hline${ }^{o} D_{7}\left(\mathrm{a}_{1}\right)$ & 4 & $19 \mathrm{~b}$ & $18 b$ & $M_{5}, M_{24}$ \\
\hline${ }^{o} D_{8}\left(\mathrm{~b}_{2}\right)$ & 24 & $19 a$ & 18a(3) & $\mathbf{M}_{\mathbf{2 4}}, M_{5}$ \\
\hline${ }^{o} D_{9}\left(\mathrm{a}_{1}\right)$ & 5 & 14 & 15 & $M_{26}, M_{25}$ \\
\hline${ }^{o} D_{10}\left(\mathrm{a}_{1}\right)$ & 6 & $7 \mathrm{a}$ & $1(20 b, 18 b, 19 b, 2,8 a, 7 a)$ & $M_{6}\left(M_{9}, M_{21}, M_{28}\right)$ \\
\hline${ }^{o} D_{11}\left(\mathrm{~b}_{2}\right)$ & 25 & 3 & $\mathbf{3}, 19 \mathrm{a}(8 \mathrm{~b})$ & $M_{25}, M_{26}\left(M_{7}, M_{28}\right)$ \\
\hline${ }^{o} D_{12}\left(\mathrm{~b}_{2}\right)$ & 26 & 13 & $12,8 b(7 b, 13,6 b)$ & $M_{6}\left(M_{9}, M_{7}, M_{21}\right)$ \\
\hline${ }^{o} D_{13}\left(\mathrm{a}_{1}\right)$ & 7 & $9 \mathrm{a}$ & $14,8 \mathrm{a}$ & $M_{27}\left(M_{7}\right)$ \\
\hline${ }^{o} D_{14}\left(\mathrm{~b}_{2}\right)$ & 27 & $18 \mathrm{a}$ & $8 b, 19 a, 12$ & $M_{7}, M_{28}\left(M_{8}, M_{9}, M_{27}\right)$ \\
\hline${ }^{o} D_{15}\left(\mathrm{a}_{1}\right)$ & 8 & $18 \mathrm{~b}$ & 19b,1(14) & $M_{8}, M_{28}\left(M_{9}\right)$ \\
\hline${ }^{o} D_{16}\left(\mathrm{~b}_{2}\right)$ & 28 & 12 & $12,6 b(7 b, 13)$ & $M_{10}, M_{9}\left(M_{29}, M_{21}\right)$ \\
\hline${ }^{o} D_{17}\left(\mathrm{a}_{1}\right)$ & 9 & 1 & $1,6 a(20 b, 19 b, 2,7 a)$ & $M_{10}\left(M_{29}, M_{9}, M_{21}\right)$ \\
\hline${ }^{o} D_{18}\left(\mathrm{a}_{1}\right)$ & 10 & $6 \mathrm{a}$ & $\mathbf{6 a}(20 \mathrm{~b})$ & $M_{29}, M_{11}$ \\
\hline${ }^{o} D_{19}\left(\mathrm{~b}_{2}\right)$ & 29 & $6 \mathrm{~b}$ & $6 b(19 a, 8 b, 3,18 a)$ & $M_{11}, M_{30}\left(M_{29}, M_{8}, M_{7}\right)$ \\
\hline${ }^{o} D_{20}\left(\mathrm{~b}_{2}\right)$ & 30 & $9 b$ & $6 b(19 a, 7 b, 13,3)$ & $M_{11}, M_{30}$ \\
\hline${ }^{o} D_{21}\left(\mathrm{a}_{1}\right)$ & 11 & 15 & $8 a, 14(19 b, 6 a)$ & $M_{30}\left(M_{7}, M_{28}, M_{8}, M_{25}\right)$ \\
\hline \multicolumn{5}{|c|}{$a^{\prime \prime}$} \\
\hline${ }^{o} D_{22}\left(\mathrm{a}_{2}\right)$ & 12 & 5 & $\mathbf{5}, 17 \mathrm{a}$ & $M_{15}\left(M_{12}\right)$ \\
\hline${ }^{o} D_{23}\left(b_{1}\right)$ & 17 & $17 \mathrm{~b}$ & $17 \mathbf{b}(10 a)$ & $M_{12}, M_{16}\left(M_{15}\right)$ \\
\hline${ }^{o} D_{24}\left(\mathrm{a}_{2}\right)$ & 13 & $17 \mathrm{a}$ & $10 b(17 a, 5)$ & $M_{16}, M_{13}\left(M_{12}\right)$ \\
\hline${ }^{o} D_{25}\left(\mathrm{~b}_{1}\right)$ & 18 & 11 & $10 \mathrm{a}, 11$ & $M_{17}\left(M_{13}, M_{18}\right)$ \\
\hline${ }^{o} D_{26}\left(\mathrm{a}_{2}\right)$ & 14 & 4 & $\mathbf{4 , 1 0 b}(17 \mathrm{a})$ & $M_{18}, M_{17}\left(M_{13}\right)$ \\
\hline${ }^{o} D_{27}\left(\mathrm{a}_{2}\right)$ & 15 & $16 \mathrm{a}$ & 16a, $4(10 b, 17 a)$ & $M_{19}\left(M_{18}, M_{14}, M_{13}\right)$ \\
\hline${ }^{o} D_{28}\left(\mathrm{~b}_{1}\right)$ & 19 & $16 \mathrm{~b}$ & $\mathbf{1 6 b}(11,10 \mathrm{a})$ & $M_{\mathbf{1 4}}, M_{19}$ \\
\hline${ }^{o} D_{29}\left(\mathrm{~b}_{1}\right)$ & 20 & $10 \mathrm{a}$ & $16 \mathrm{~b}, 11(10 \mathrm{a})$ & $\boldsymbol{M}_{\mathbf{2 0}}\left(M_{14}, M_{19}\right)$ \\
\hline${ }^{o} D_{30}\left(\mathrm{a}_{2}\right)$ & 16 & $10 \mathrm{~b}$ & $16 a(17 a, 5,10 b, 4)$ & $M_{20}\left(M_{14}, M_{13}, M_{16}\right)$ \\
\hline
\end{tabular}

a This work - see text. Labels based on Mulliken numbering in $C_{s}$ symmetry.

b Mulliken numbering in $C_{2 v}$ symmetry.

${ }^{c}$ It should be noted that we have highlighted in Ref. [2] that several Wilson modes have been misnumbered in various texts over the years, with the following switches often being required: $8 \mathrm{a} \leftrightarrow 9 \mathrm{a}, 8 \mathrm{~b} \leftrightarrow 9 \mathrm{~b}, 18 \mathrm{a} \leftrightarrow 19 \mathrm{a}, 18 \mathrm{~b} \leftrightarrow 19 \mathrm{~b}$ and $3 \leftrightarrow 14$; where they have occurred, these misnumberings have been corrected in the present work. For example, such misnumberings are present in the work of Varsányi [3] and these have been carried forward into other work, such as Ref. [26].

${ }^{\mathrm{d}}$ These express the $\mathrm{S}_{0}$ oDFB ${ }^{o} D_{i}$ modes in terms of those of the benzene Wilson modes using a generalized Duschinsky approach involving artificial isotopologues - see text and Ref. [11]. Values outside parentheses have mixing coefficients $>0.2$ and are termed major contributions, with bolded values being dominant contributions (mixing coefficients $>0.5$ ). Those inside parentheses are minor contributions, and have values between 0.05 and 0.2 . 
If there is more than one contribution of each type, these are given in numerical order. Vibrations with a mixing coefficient $<0.05$ are ignored.

e These express the $\mathrm{S}_{0} o \mathrm{DFB}^{o} D_{i}$ modes in terms of those of fluorobenzene, numbered as $M_{i}$ modes (see ref. [2]), using a generalized Duschinsky approach involving artificial isotopologues - see text and Ref. [11]. Values outside parentheses have mixing coefficients $>0.2$ and are termed major contributions, with bolded values being dominant contributions (mixing coefficients $>0.5$ ). Those inside parentheses are minor contributions, and have values between 0.05 and 0.2 . If there is more than one contribution of each type, these are given in numerical order. Vibrations with a mixing coefficient $<0.05$ are ignored. 
Table 2: Vibrational wavenumbers for $o-\mathrm{C}_{6} \mathrm{H}_{4} \mathrm{X}_{2}\left(\mathrm{X}=\mathrm{F}, \mathrm{Cl}, \mathrm{Br}, \mathrm{I}, \mathrm{CH}_{3}\right.$ and $\left.\mathrm{OH}\right)$

\begin{tabular}{|c|c|c|c|c|c|c|c|c|c|c|c|c|c|}
\hline \multicolumn{2}{|c|}{ Mode } & \multicolumn{2}{|c|}{$o \mathrm{Xyl}$} & \multicolumn{2}{|c|}{ Catechol } & \multicolumn{2}{|c|}{$o \mathrm{DFB}$} & \multicolumn{2}{|c|}{$o \mathrm{DClB}$} & \multicolumn{2}{|c|}{$o \mathrm{DBrB}$} & \multicolumn{2}{|c|}{$o \mathrm{DIB}$} \\
\hline$D_{i}$ & $C_{2 v}$ & $\operatorname{Expt}^{\mathrm{a}}$ & $\mathrm{Calc}^{\mathrm{b}}$ & $\operatorname{Expt}^{\mathrm{c}}$ & $\mathrm{Calc}^{\mathrm{b}}$ & $\operatorname{Expt}^{\mathrm{a}, \mathrm{d}}$ & $\mathrm{Calc}^{\mathrm{b}}$ & $\operatorname{Expt}^{\mathrm{a}}$ & $\mathrm{Calc}^{\mathrm{b}}$ & $\operatorname{Expt}^{\mathrm{a}}$ & $\mathrm{Calc}^{\mathrm{b}}$ & $\operatorname{Expt}^{\mathrm{a}}$ & $\mathrm{Calc}^{\mathrm{b}}$ \\
\hline \multicolumn{14}{|c|}{$a^{\prime}$} \\
\hline${ }^{\circ} D_{1}\left(a_{1}\right)$ & 1 & 3080 & 3092 & $3081^{\mathrm{e}, \mathrm{g}}$ & 3104 & 3081 & 3110 & 3072 & 3111 & 3067 & 3110 & 3065 & 3108 \\
\hline${ }^{\circ} D_{2}\left(b_{2}\right)$ & 21 & 3080 & 3077 & $3060^{\mathrm{h}}$ & 3095 & 3081 & 3104 & 3072 & 3108 & 3067 & 3106 & 3065 & 3104 \\
\hline${ }^{\circ} D_{3}\left(a_{1}\right)$ & 2 & 3064 & 3063 & $3051^{\mathrm{h}}$ & 3082 & 3045 & 3096 & - & 3096 & - & 3095 & 3052 & 3092 \\
\hline${ }^{\circ} D_{4}\left(b_{2}\right)$ & 22 & 3048 & 3059 & $3051^{\mathrm{h}}$ & 3059 & 3060 & 3085 & - & 3083 & - & 3083 & 3052 & 3079 \\
\hline${ }^{\circ} D_{5}\left(a_{1}\right)$ & 3 & - & 1568 & $1616^{\mathrm{h}}$ & 1603 & $1625^{\mathrm{d}}$ & 1596 & 1576 & 1559 & 1567 & 1554 & 1556 & 1547 \\
\hline${ }^{\circ} D_{6}\left(b_{2}\right)$ & 23 & - & 1596 & $1607^{\mathrm{h}}$ & 1596 & $1619^{d}$ & 1591 & 1576 & 1565 & 1567 & 1557 & 1556 & 1550 \\
\hline${ }^{\circ} D_{7}\left(\mathrm{a}_{1}\right)$ & 4 & 1494 & 1480 & $1504^{\mathrm{h}}$ & 1498 & $1507^{d}$ & 1495 & 1458 & 1446 & 1449 & 1437 & 1439 & 1429 \\
\hline${ }^{\circ} D_{8}\left(b_{2}\right)$ & 24 & 1468 & 1459 & $1479^{\mathrm{h}}$ & 1463 & 1472 & 1448 & 1438 & 1422 & 1431 & 1417 & 1425 & 1409 \\
\hline${ }^{\circ} D_{9}\left(a_{1}\right)$ & 5 & 1292 & 1285 & $1324^{\mathrm{g}}$ & 1320 & $1299^{d}$ & 1292 & 1276 & 1267 & $1268^{\mathrm{e}}$ & 1261 & $1260^{\mathrm{m}}$ & 1249 \\
\hline${ }^{o} D_{10}\left(\mathrm{a}_{1}\right)$ & 6 & 1222 & $\begin{array}{c}1205 \\
\text { (sym) }\end{array}$ & $1263^{\mathrm{e}, \mathrm{g}}$ & $\begin{array}{c}1259 \\
(\mathrm{sym})\end{array}$ & $1279^{d}$ & $\begin{array}{c}1254 \\
\text { (sym) }\end{array}$ & $1130^{\mathrm{k}}$ & $\begin{array}{c}1105 \\
(\mathrm{sym})\end{array}$ & $1106^{\mathrm{k}}$ & $\begin{array}{c}1084 \\
(\mathrm{sym})\end{array}$ & $1086^{\mathrm{k}}$ & $\begin{array}{c}1070 \\
\text { (sym) }\end{array}$ \\
\hline${ }^{\circ} D_{11}\left(b_{2}\right)$ & 25 & 1290 & 1281 & $1251^{\mathrm{h}}$ & 1229 & 1253 & 1252 & 1252 & 1241 & 1255 & 1241 & 1255 & 1243 \\
\hline${ }^{o} D_{12}\left(b_{2}\right)$ & 26 & 1185 & $\begin{array}{l}1169 \\
\text { (asym) }\end{array}$ & $1151^{\mathrm{i}, \mathrm{j}}$ & $\begin{array}{l}1133 \\
\text { (asym) }\end{array}$ & 1206 & $\begin{array}{l}1182 \\
\text { (asym) }\end{array}$ & $1038^{1}$ & $\begin{array}{l}1015 \\
\text { (asym) }\end{array}$ & $1012^{1}$ & $\begin{array}{c}995 \\
\text { (asym) }\end{array}$ & $990^{1}$ & $\begin{array}{c}980 \\
\text { (asym) }\end{array}$ \\
\hline${ }^{o} D_{13}\left(a_{1}\right)$ & 7 & 1155 & 1149 & $1151^{\mathrm{h}}$ & 1142 & $1153^{\mathrm{d}}$ & 1141 & $1155^{\mathrm{k}}$ & 1149 & $1159^{\mathrm{k}}$ & 1150 & $1160^{\mathrm{k}}$ & 1153 \\
\hline${ }^{\circ} D_{14}\left(b_{2}\right)$ & 27 & 1121 & 1111 & $1092^{\mathrm{h}}$ & 1076 & 1101 & 1090 & $1130^{1}$ & 1115 & $1107^{1}$ & 1109 & $1100^{1}$ & 1104 \\
\hline${ }^{\circ} D_{15}\left(a_{1}\right)$ & 8 & 1052 & 1042 & $1030^{\mathrm{g}}$ & 1021 & $1027^{d}$ & 1017 & $1040^{\mathrm{e}}$ & 1026 & 1035 & 1023 & 1029 & 1016 \\
\hline${ }^{\circ} D_{16}\left(b_{2}\right)$ & 28 & 826 & 813 & $859^{\mathrm{h}}$ & 839 & 856 & 838 & 740 & 726 & 700 & 690 & 672 & 669 \\
\hline${ }^{o} D_{17}\left(a_{1}\right)$ & 9 & $733^{e}$ & 724 & $768^{\mathrm{h}}$ & 757 & $766^{d}$ & 752 & 660 & 652 & 640 & 635 & 631 & 626 \\
\hline${ }^{o} D_{18}\left(a_{1}\right)$ & 10 & 581 & 574 & $564^{g}$ & 574 & $568^{d}$ & 565 & 480 & 464 & 375 & 364 & 317 & 308 \\
\hline${ }^{\circ} D_{19}\left(b_{2}\right)$ & 29 & 505 & 495 & $542^{g}$ & 542 & 546 & 536 & 427 & 415 & $358^{\mathrm{e}}$ & 348 & $317^{\mathrm{n}}$ & 308 \\
\hline${ }^{\circ} D_{20}\left(b_{2}\right)$ & 30 & 406 & 399 & - & 435 & $438^{\mathrm{d}}$ & 430 & 336 & 327 & 251 & 244 & $206^{n}$ & 201 \\
\hline${ }^{o} D_{21}\left(a_{1}\right)$ & 11 & 255 & 291 & $320^{\mathrm{g}}$ & 299 & $286^{\mathrm{d}}$ & 279 & 202 & 193 & 129 & 123 & 98 & 92 \\
\hline \multicolumn{14}{|c|}{$a^{\prime \prime}$} \\
\hline${ }^{o} D_{22}\left(a_{2}\right)$ & 12 & {$[970]^{\mathrm{f}}$} & 976 & $963^{g}$ & 954 & 970 & 966 & 975 & 972 & {$[975]^{\mathrm{f}}$} & 975 & {$[970]^{\mathrm{f}}$} & 977 \\
\hline${ }^{\circ} D_{23}\left(b_{1}\right)$ & 17 & 930 & 932 & $916^{\mathrm{h}}$ & 912 & $929^{d}$ & 930 & 940 & 943 & 940 & 944 & 939 & 946 \\
\hline${ }^{\circ} D_{24}\left(a_{2}\right)$ & 13 & 860 & 861 & $851^{\mathrm{g}}$ & 836 & $850^{\mathrm{d}}$ & 847 & 850 & 854 & 854 & 854 & 855 & 857 \\
\hline${ }^{o} D_{25}\left(b_{1}\right)$ & 18 & 741 & 739 & $741^{\mathrm{h}}$ & 734 & 749 & 748 & 748 & 747 & 745 & 745 & 745 & 743 \\
\hline${ }^{\circ} D_{26}\left(a_{2}\right)$ & 14 & $702^{\mathrm{e}}$ & 713 & $721^{g}$ & 724 & $705^{\mathrm{d}}$ & 703 & $695^{\mathrm{e}}$ & 689 & {$[695]^{\mathrm{f}}$} & 680 & {$[695]^{\mathrm{f}}$} & 687 \\
\hline${ }^{\circ} D_{27}\left(a_{2}\right)$ & 15 & {$[505]^{\mathrm{f}}$} & 511 & $582^{\mathrm{g}}$ & 559 & $556^{\mathrm{d}}$ & 549 & $504^{\mathrm{e}}$ & 509 & 494 & 496 & 478 & 482 \\
\hline${ }^{\circ} D_{28}\left(b_{1}\right)$ & 19 & 435 & 438 & $456^{\mathrm{g}}$ & 451 & $452^{\mathrm{d}}$ & 452 & 435 & 438 & 431 & 431 & 420 & 425 \\
\hline${ }^{\circ} D_{29}\left(b_{1}\right)$ & 20 & 325 & 251 & $299^{j}$ & 289 & 275 & 284 & 239 & 228 & $217^{e}$ & 206 & 200 & 187 \\
\hline${ }^{\circ} D_{30}\left(a_{2}\right)$ & 16 & 180 & 171 & $198^{\mathrm{g}}$ & 185 & $186^{\mathrm{d}}$ & 183 & 152 & 132 & $136^{\mathrm{e}}$ & 112 & 124 & 100 \\
\hline
\end{tabular}

${ }^{a}$ Infrared spectroscopy of the liquid unless otherwise indicated, from Ref. [20].

${ }^{\mathrm{b}}$ B3LYP/aug-cc-pVTZ values scaled by 0.97 - this work (see text). For consistency with the following tables, we have noted the symmetric and asymmetric stretch nature of $D_{10}$ and $D_{12}$ explicitly. The conformer used for $o$ Xyl was trans eclipsed-eclipsed, where one $\mathrm{CH}$ bond from each methyl is in the plane of the phenyl ring, and these are oriented away from each other. The conformer used for catechol was the expected lowest energy one, consistent with other work, with the OH groups parallel and hydrogen bonded.

${ }^{\mathrm{c}}$ Experimental values taken from Refs. [27] and [31], favouring the latter, more-recent work.

${ }^{\mathrm{d}}$ A number of experimental values were derived by us from assignments and band positions given in the jet-cooled dispersed fluorescence study [26].

e Raman studies of liquid [25].

${ }^{\mathrm{f}}$ Estimated value from Ref. [20].

g Infrared and Raman values summarized in a surface-enhanced Raman spectroscopy study by Koh et al. [31].

h Value for vapour [29].

${ }^{i}$ We favour the given value based on the calculated value and its motion, although the assignment was not given in Ref. [27].

j From a summary of values used in thermochemical study by Kudchadker et al. [27].

${ }^{\mathrm{k}}$ Wavenumber ordering has been switched from that in Ref. [20].

${ }^{1}$ Wavenumber ordering has been switched from Ref. [20]. 
${ }^{m}$ Raman studies of liquid [24]

${ }^{\mathrm{n}}$ Reassigned from Ref. [20] - see text. 
Table 3: Vibrational wavenumbers for $o-\mathrm{C}_{6} \mathrm{H}_{4} \mathrm{XY}$ : asymmetric ortho-dihalobenzenes

\begin{tabular}{|c|c|c|c|c|c|c|c|c|c|c|c|c|}
\hline \multirow{2}{*}{ Mode } & \multicolumn{2}{|c|}{$o \mathrm{ClFB}$} & \multicolumn{2}{|c|}{$o \mathrm{BrFB}$} & \multicolumn{2}{|c|}{$o$ FIB } & \multicolumn{2}{|c|}{$o \mathrm{BrClB}$} & \multicolumn{2}{|c|}{$o$ ClIB } & \multicolumn{2}{|c|}{ oBrIB } \\
\hline & $\operatorname{Expt}^{\mathrm{a}}$ & Calc $^{b}$ & $\operatorname{Expt}^{\mathrm{a}}$ & $\mathrm{Calc}^{\mathrm{b}}$ & $\operatorname{Expt}^{\mathrm{a}}$ & Calc $^{\mathrm{b}}$ & $\operatorname{Expt}^{\mathrm{a}}$ & Calc $^{\mathrm{b}}$ & $\operatorname{Expt}^{\mathrm{a}}$ & Calc $^{\mathrm{b}}$ & $\operatorname{Expt}^{\mathrm{a}}$ & Calc $^{\mathrm{b}}$ \\
\hline \multicolumn{13}{|c|}{$a^{\prime}$} \\
\hline$D_{1}$ & $3082^{c}$ & 3110 & 3078 & 3109 & 3074 & 3108 & 3086 & 3110 & 3088 & 3109 & 3078 & 3109 \\
\hline$D_{2}$ & 3062 & 3105 & 3065 & 3105 & 3051 & 3104 & 3067 & 3106 & 3062 & 3105 & 3059 & 3105 \\
\hline$D_{3}$ & - & 3096 & - & 3095 & - & 3094 & - & 3095 & - & 3094 & - & 3094 \\
\hline$D_{4}$ & - & 3085 & - & 3084 & - & 3083 & - & 3082 & - & 3081 & - & 3081 \\
\hline$D_{5}$ & 1597 & 1579 & 1592 & 1575 & 1582 & 1571 & 1572 & 1556 & 1566 & 1553 & 1562 & 1550 \\
\hline$D_{6}$ & 1588 & 1575 & 1580 & 1572 & 1572 & 1568 & 1565 & 1562 & 1566 & 1557 & 1562 & 1554 \\
\hline$D_{7}$ & 1481 & 1470 & 1480 & 1465 & 1472 & 1459 & 1458 & 1442 & 1447 & 1437 & 1441 & 1433 \\
\hline$D_{8}$ & 1450 & 1436 & 1448 & 1433 & 1442 & 1429 & 1435 & 1419 & 1425 & 1415 & 1424 & 1412 \\
\hline$D_{9}$ & 1288 & 1283 & 1287 & 1281 & 1280 & 1278 & $1269^{\mathrm{d}}$ & 1264 & 1267 & 1259 & $1260^{\mathrm{d}}$ & 1255 \\
\hline$D_{10}$ & $1240^{\mathrm{e}}$ & $\begin{array}{l}1221 \\
\text { (C-F) }\end{array}$ & $1235^{\mathrm{e}}$ & $\begin{array}{l}1219 \\
\text { (C-F) }\end{array}$ & $1233^{\mathrm{e}}$ & $\begin{array}{l}1215 \\
\text { (C-F) }\end{array}$ & $1115^{\mathrm{e}}$ & $\begin{array}{c}1094 \\
\text { (sym) }\end{array}$ & $1103^{\mathrm{e}}$ & $\begin{array}{c}1085 \\
\text { (sym) }\end{array}$ & $1094^{\mathrm{e}}$ & $\begin{array}{c}1076 \\
\text { (sym) }\end{array}$ \\
\hline$D_{11}$ & $1266^{\mathrm{e}}$ & 1250 & $1263^{\mathrm{e}}$ & 1249 & $1261^{\mathrm{e}}$ & 1247 & $1252^{\mathrm{e}}$ & 1241 & $1256^{\mathrm{e}}$ & 1241 & $1253^{\mathrm{e}}$ & 1241 \\
\hline$D_{12}$ & $1068^{\mathrm{e}}$ & $\begin{array}{c}1048 \\
(\mathrm{C}-\mathrm{Cl}) \\
\end{array}$ & $1050^{\mathrm{e}}$ & $\begin{array}{c}1034 \\
\text { (C-Br) }\end{array}$ & $1042^{\mathrm{e}}$ & $\begin{array}{l}1010 \\
(\mathrm{C}-\mathrm{I}) \\
\end{array}$ & $1023^{\mathrm{e}}$ & $\begin{array}{c}1004 \\
\text { (asym) }\end{array}$ & $1013^{\mathrm{e}}$ & $\begin{array}{c}995 \\
\text { (asym) }\end{array}$ & $1003^{\mathrm{e}}$ & $\begin{array}{c}988 \\
\text { (asym) } \\
\end{array}$ \\
\hline$D_{13}$ & $1158^{\mathrm{e}}$ & 1145 & $1158^{\mathrm{e}}$ & 1145 & $1160^{\mathrm{e}}$ & 1145 & $1160^{\mathrm{e}}$ & 1150 & $1158^{\mathrm{e}}$ & 1150 & $1160^{\mathrm{e}}$ & 1151 \\
\hline$D_{14}$ & $1126^{\mathrm{e}}$ & 1110 & $1117^{\mathrm{e}}$ & 1105 & $1113^{\mathrm{e}}$ & 1101 & $1124^{\mathrm{e}}$ & 1114 & $1116^{\mathrm{e}}$ & 1111 & $1105^{\mathrm{e}}$ & 1107 \\
\hline$D_{15}$ & 1031 & 1020 & 1026 & 1017 & 1022 & 1029 & $1036^{\mathrm{e}}$ & 1025 & $1035^{\mathrm{e}}$ & 1024 & $1031^{\mathrm{e}}$ & 1020 \\
\hline$D_{16}$ & 826 & 813 & 820 & 810 & 819 & 808 & 719 & 710 & 717 & 703 & 686 & 680 \\
\hline$D_{17}$ & 681 & 669 & 650 & 647 & 640 & 637 & 646 & 640 & 636 & 633 & 633 & 639 \\
\hline$D_{18}$ & 553 & 541 & 544 & 533 & 540 & 529 & 444 & 434 & $-^{f}$ & 425 & 354 & 342 \\
\hline$D_{19}$ & 496 & 483 & 472 & 460 & 462 & 451 & 387 & 375 & 243 & 358 & 222 & 328 \\
\hline$D_{20}$ & 375 & 366 & 297 & 289 & 253 & 246 & 282 & 274 & 224 & 237 & 209 & 219 \\
\hline$D_{21}$ & 229 & 221 & 189 & 181 & 165 & 159 & 164 & 157 & 146 & 139 & 113 & 107 \\
\hline \multicolumn{13}{|c|}{$a^{\prime \prime}$} \\
\hline$D_{22}$ & 974 & 972 & 970 & 973 & 980 & 973 & 980 & 977 & 976 & 978 & 975 & 978 \\
\hline$D_{23}$ & 934 & 935 & 938 & 937 & 942 & 938 & 940 & 944 & 941 & 945 & 941 & 944 \\
\hline$D_{24}$ & 850 & 848 & 851 & 848 & 851 & 848 & 854 & 853 & 855 & 855 & $854^{\mathrm{g}}$ & 852 \\
\hline$D_{26}$ & 749 & 749 & 751 & 750 & 751 & 750 & 746 & 747 & 745 & 746 & 744 & 744 \\
\hline$D_{25}$ & 698 & 701 & 694 & 697 & 694 & 690 & 689 & 686 & 689 & 682 & {$[685]^{\mathrm{h}}$} & 677 \\
\hline$D_{27}$ & 535 & 534 & 530 & 530 & 527 & 525 & 501 & 503 & 495 & 497 & 484 & 489 \\
\hline$D_{28}$ & 443 & 442 & 439 & 439 & 437 & 435 & 433 & 435 & 430 & 430 & 426 & 427 \\
\hline$D_{29}$ & 268 & 260 & 261 & 253 & 253 & 247 & 230 & 218 & 213 & 209 & 209 & 196 \\
\hline$D_{30}$ & 168 & 152 & 155 & 137 & 143 & 125 & 140 & 122 & 132 & 112 & 127 & 105 \\
\hline
\end{tabular}

${ }^{a}$ Infrared spectroscopy of the liquid unless otherwise indicated, from Ref. [20].

${ }^{\mathrm{b}}$ B3LYP/aug-cc-pVTZ values scaled by 0.97 - this work (see text). We have noted the symmetric and asymmetric stretch, or the localized nature, of $D_{10}$ and $D_{12}$ explicitly.

${ }^{\mathrm{c}}$ Raman studies of liquid [33].

${ }^{\mathrm{d}}$ Raman studies of liquid [24].

${ }^{\text {e }}$ Note that some or all of the vibrations $D_{10}-D_{15}$ are not in the same wavenumber order as in $o \mathrm{DFB}$, but vibrations with similar motions have been given the same $D_{i}$ label. The experimental wavenumbers have been ordered to match the calculated ordering.

${ }^{\mathrm{f}}$ A value of $366 \mathrm{~cm}^{-1}$ was given for this vibration in Ref. [20], but this appears to be an incorrect assignment - see text. 
g Value mistyped in Ref. [20]; this value is consistent with the values for the other species and a Raman value cited in that work.

${ }^{\mathrm{h}}$ Estimated value from Ref. [20]. 
Table 4: Vibrational wavenumbers for $o$-halotoluenes ${ }^{\mathrm{a}}$

\begin{tabular}{|c|c|c|c|c|c|c|c|c|}
\hline \multirow[t]{2}{*}{ Mode } & \multicolumn{2}{|c|}{$o \mathrm{FT}$} & \multicolumn{2}{|c|}{$o \mathrm{ClT}$} & \multicolumn{2}{|c|}{$o \mathrm{BrT}$} & \multicolumn{2}{|c|}{$o \mathrm{IT}$} \\
\hline & $\operatorname{Expt}^{\mathrm{a}}$ & $\mathrm{Calc}^{\mathrm{b}}$ & Expt $^{\mathrm{a}}$ & $\mathrm{Calc}^{\mathrm{b}}$ & Expt $^{\mathrm{a}}$ & $\mathrm{Calc}^{\mathrm{b}}$ & Expt $^{\mathrm{a}}$ & $\mathrm{Calc}^{\mathrm{b}}$ \\
\hline \multicolumn{9}{|c|}{$a^{\prime}$} \\
\hline$D_{1}$ & 3067 & 3105 & 3067 & 3107 & 3060 & 3106 & 3060 & 3103 \\
\hline$D_{2}$ & 3045 & 3082 & 3057 & 3081 & - & 3081 & - & 3080 \\
\hline$D_{3}$ & - & 3095 & - & 3095 & - & 3094 & - & 3093 \\
\hline$D_{4}$ & - & 3069 & - & 3069 & - & 3068 & - & 3067 \\
\hline$D_{5}$ & 1621 & 1574 & 1592 & 1560 & 1592 & 1556 & 1590 & 1553 \\
\hline$D_{6}$ & 1589 & 1603 & 1572 & 1585 & 1565 & 1582 & 1562 & 1576 \\
\hline$D_{7}$ & 1490 & 1482 & 1473 & 1465 & 1467 & 1462 & 1462 & 1459 \\
\hline$D_{8}$ & 1467 & 1429 & 1458 & 1419 & 1456 & 1417 & 1453 & 1415 \\
\hline$D_{9}$ & 1299 & 1293 & 1282 & 1277 & 1274 & 1273 & 1274 & 1268 \\
\hline$D_{10}$ & $1233^{c}$ & $\begin{array}{l}1216 \\
(\mathrm{sym})\end{array}$ & $\begin{array}{l}1054^{\mathrm{c}} \\
(\mathrm{C}-\mathrm{Cl})\end{array}$ & $\begin{array}{c}1028 \\
(\mathrm{C}-\mathrm{Cl})\end{array}$ & $\begin{array}{l}1031^{\mathrm{c}} \\
(\mathrm{C}-\mathrm{Br})\end{array}$ & $\begin{array}{c}1009 \\
(\mathrm{C}-\mathrm{Br})\end{array}$ & $\begin{array}{l}1016^{c} \\
(\mathrm{C}-\mathrm{I})\end{array}$ & $\begin{array}{l}997 \\
(\mathrm{C}-\mathrm{I})\end{array}$ \\
\hline$D_{11}$ & $1267^{c}$ & 1268 & $1253^{c}$ & 1266 & $1252^{\mathrm{c}}$ & 1266 & {$[1250]^{\mathrm{c}, \mathrm{e}}$} & 1265 \\
\hline$D_{12}$ & 1187 & $\begin{array}{c}1169 \\
\text { (asym) }\end{array}$ & $\begin{array}{c}1207^{\mathrm{c}} \\
(\mathrm{C}-\mathrm{Me})\end{array}$ & $\begin{array}{c}1192 \\
(\mathrm{C}-\mathrm{Me})\end{array}$ & $\begin{array}{c}1208^{\mathrm{c}} \\
(\mathrm{C}-\mathrm{Me})\end{array}$ & $\begin{array}{c}1193 \\
(\mathrm{C}-\mathrm{Me})\end{array}$ & $\begin{array}{c}1206^{\mathrm{c}} \\
(\mathrm{C}-\mathrm{Me})\end{array}$ & $\begin{array}{c}1192 \\
(\mathrm{C}-\mathrm{Me})\end{array}$ \\
\hline$D_{13}$ & 1147 & 1143 & $1158^{\mathrm{c}}$ & 1148 & $1158^{c}$ & 1149 & $1157^{\mathrm{c}}$ & 1150 \\
\hline$D_{14}$ & 1112 & 1100 & $1129^{c}$ & 1120 & $1120^{c}$ & 1117 & $1117^{\mathrm{c}}$ & 1114 \\
\hline$D_{15}$ & 1037 & 1028 & 1042 & 1037 & $1045^{c}$ & 1037 & $1046^{c}$ & 1038 \\
\hline$D_{16}$ & 840 & 823 & 805 & 790 & 798 & 786 & 795 & 784 \\
\hline$D_{17}$ & $747^{d}$ & 737 & 678 & 669 & 656 & 650 & 643 & 642 \\
\hline$D_{18}$ & 576 & 568 & 553 & 541 & 543 & 533 & 538 & 529 \\
\hline$D_{19}$ & 525 & 516 & 445 & 432 & $411^{\mathrm{d}}$ & 400 & 405 & 391 \\
\hline$D_{20}$ & 426 & 422 & 366 & 359 & 297 & 286 & 250 & 244 \\
\hline$D_{21}$ & 272 & 268 & 246 & 239 & $215^{\mathrm{d}}$ & 201 & 193 & 182 \\
\hline \multicolumn{9}{|c|}{$a^{\prime \prime}$} \\
\hline$D_{22}$ & {$[970]^{\mathrm{e}}$} & 973 & 970 & 978 & 970 & 978 & 970 & 978 \\
\hline$D_{23}$ & 935 & 934 & 935 & 939 & 935 & 938 & 936 & 939 \\
\hline$D_{24}$ & $855^{f}$ & 850 & 854 & 854 & 855 & 852 & 855 & 853 \\
\hline$D_{25}$ & 754 & 752 & 747 & 746 & 744 & 743 & 743 & 741 \\
\hline$D_{26}$ & 704 & 707 & 701 & 704 & 694 & 701 & 693 & 699 \\
\hline$D_{27}$ & 536 & 538 & 508 & 513 & 501 & 505 & 494 & 498 \\
\hline$D_{28}$ & 441 & 442 & 436 & 437 & 433 & 434 & 430 & 430 \\
\hline$D_{29}$ & 288 & 262 & 246 & 237 & 238 & 229 & 231 & 223 \\
\hline$D_{30}$ & 186 & 174 & 164 & 151 & 157 & 131 & 148 & 124 \\
\hline
\end{tabular}

${ }^{a}$ Infrared spectroscopy of the liquid unless otherwise indicated, from Ref. [20].

${ }^{\mathrm{b}}$ B3LYP/aug-cc-pVTZ values scaled by 0.97 - this work (see text). The conformer in each case has the methyl group eclipsed (one $\mathrm{C}-\mathrm{H}$ bond in the plane of the phenyl ring) and pointing away from the halogen atom. We have noted the symmetric and asymmetric stretch, or the localized nature, of $D_{10}$ and $D_{12}$ explicitly (where C-Me denotes a carbonmethyl bond).

${ }^{c}$ Note that some or all of the vibrations $D_{10}-D_{15}$ are not in the same wavenumber order as in $o \mathrm{DFB}$, but vibrations with similar motions have been given the same $D_{i}$ label. The experimental wavenumbers have been ordered to match the calculated ordering.

${ }^{\mathrm{d}}$ Raman studies of liquid [25]. 
${ }^{\text {e }}$ Estimated value from Ref. [20].

${ }^{\mathrm{f}}$ A value of $955 \mathrm{~cm}^{-1}$ is given in Table 4 of Ref. [20], but this appears to be a typographical error based on the quoted Raman value therein, and has been corrected here. 
Table 5: Vibrational wavenumbers for $o$-halophenols and $o$-cresol a

\begin{tabular}{|c|c|c|c|c|c|c|c|c|c|c|}
\hline \multirow[t]{2}{*}{ Mode } & \multicolumn{2}{|c|}{$o$-cresol } & \multicolumn{2}{|c|}{$o \mathrm{FPhOH}$} & \multicolumn{2}{|c|}{$o \mathrm{ClPhOH}$} & \multicolumn{2}{|c|}{$o \mathrm{BrPhOH}$} & \multicolumn{2}{|c|}{$o \mathrm{IPhOH}$} \\
\hline & Expt $^{a}$ & $\mathrm{Calc}^{\mathrm{b}}$ & $\operatorname{Expt}^{\mathrm{a}}$ & $\mathrm{Calc}^{\mathrm{b}}$ & Expt $^{a}$ & $\mathrm{Calc}^{\mathrm{b}}$ & $\operatorname{Expt}^{\mathrm{a}}$ & $\mathrm{Calc}^{\mathrm{b}}$ & $\operatorname{Expt}^{\mathrm{a}}$ & $\mathrm{Calc}^{\mathrm{b}}$ \\
\hline \multicolumn{11}{|c|}{$a^{\prime}$} \\
\hline$D_{1}$ & $3060^{c}$ & 3096 & $3062^{\mathrm{c}}$ & 3108 & $3085^{c}$ & 3109 & $3076^{c}$ & 3108 & $3071^{c}$ & 3106 \\
\hline$D_{2}$ & $3049^{\mathrm{c}, \mathrm{d}}$ & 3081 & $\mathrm{c}$ & 3100 & $3060^{\mathrm{d}}$ & 3101 & $3035^{c}$ & 3100 & $3043^{c}$ & 3099 \\
\hline$D_{3}$ & $3028^{c}$ & 3065 & c & 3093 & c & 3094 & c & 3093 & $3026^{c}$ & 3091 \\
\hline$D_{4}$ & $--^{c}$ & 3053 & c & 3081 & $\mathrm{c}$ & 3081 & $\mathrm{c}$ & 3080 & $\mathrm{c}$ & 3079 \\
\hline$D_{5}$ & $1587^{\mathrm{e}}$ & 1583 & 1616 & 1602 & 1584 & 1575 & 1586 & 1570 & 1584 & 1565 \\
\hline$D_{6}$ & $1608^{\mathrm{e}}$ & 1599 & 1599 & 1592 & 1595 & 1584 & 1599 & 1581 & 1591 & 1576 \\
\hline$D_{7}$ & 1492 & 1492 & 1513 & 1489 & 1480 & 1468 & 1476 & 1464 & 1472 & 1459 \\
\hline$D_{8}$ & 1462 & 1429 & 1461 & 1459 & 1461 & 1449 & 1449 & 1446 & 1445 & 1442 \\
\hline$D_{9}$ & 1324 & 1317 & 1349 & 1342 & 1337 & 1330 & 1334 & 1327 & 1333 & 1324 \\
\hline$D_{10}$ & $\begin{array}{c}1255^{\mathrm{e}} \\
(\mathrm{C}-\mathrm{OH})\end{array}$ & $\begin{array}{c}1239 \\
(\mathrm{C}-\mathrm{OH})\end{array}$ & $\begin{array}{l}1262^{\mathrm{e}} \\
(\mathrm{sym})\end{array}$ & $\begin{array}{l}1249 \\
\text { (sym) }\end{array}$ & $\begin{array}{c}1252^{\mathrm{e}} \\
(\mathrm{C}-\mathrm{OH})\end{array}$ & $\begin{array}{c}1239 \\
(\mathrm{C}-\mathrm{OH})\end{array}$ & $\begin{array}{c}1249^{\mathrm{e}} \\
(\mathrm{C}-\mathrm{OH})\end{array}$ & $\begin{array}{c}1238 \\
(\mathrm{C}-\mathrm{OH})\end{array}$ & $\begin{array}{c}1246^{\mathrm{e}} \\
(\mathrm{C}-\mathrm{OH})\end{array}$ & $\begin{array}{c}1235 \\
(\mathrm{C}-\mathrm{OH})\end{array}$ \\
\hline$D_{11}$ & $1300^{\mathrm{e}}$ & 1292 & $1292^{\mathrm{e}}$ & 1286 & $1294^{\mathrm{e}}$ & 1285 & $1292^{\mathrm{e}}$ & 1285 & $1293^{\mathrm{e}}$ & 1283 \\
\hline$D_{12}$ & $\begin{array}{c}1207 \\
(\mathrm{C}-\mathrm{Me})\end{array}$ & $\begin{array}{c}1197 \\
(\mathrm{C}-\mathrm{Me})\end{array}$ & $\begin{array}{c}1218 \\
\text { (asym) }\end{array}$ & $\begin{array}{c}1201 \\
\text { (asym) }\end{array}$ & $\begin{array}{l}1057^{\mathrm{e}} \\
(\mathrm{C}-\mathrm{Cl})\end{array}$ & $\begin{array}{c}1015 \\
(\mathrm{C}-\mathrm{Cl})\end{array}$ & $\begin{array}{c}1040^{\mathrm{e}} \\
(\mathrm{C}-\mathrm{Br})\end{array}$ & $\begin{array}{c}1003 \\
(\mathrm{C}-\mathrm{Br})\end{array}$ & $\begin{array}{c}1039^{\mathrm{e}} \\
(\mathrm{C}-\mathrm{I})\end{array}$ & $\begin{array}{l}994 \\
(\mathrm{C}-\mathrm{I})\end{array}$ \\
\hline$D_{13}$ & 1149 & 1144 & 1152 & 1141 & $1154^{\mathrm{e}}$ & 1144 & $1153^{\mathrm{e}}$ & 1144 & $1154^{\mathrm{e}}$ & 1145 \\
\hline$D_{14}$ & 1102 & 1089 & 1092 & 1078 & $1124^{\mathrm{e}}$ & 1108 & $1114^{\mathrm{e}}$ & 1103 & $1108^{\mathrm{e}}$ & 1099 \\
\hline$D_{15}$ & 1043 & 1036 & 1023 & 1015 & 1028 & 1032 & 1022 & 1028 & 1021 & 1029 \\
\hline$D_{16}$ & 842 & 828 & 857 & 837 & 835 & 821 & 829 & 818 & 827 & 817 \\
\hline$D_{17}$ & 748 & 738 & 763 & 753 & 677 & 665 & 654 & 646 & 641 & 638 \\
\hline$D_{18}$ & 586 & 575 & 570 & 568 & 557 & 548 & 547 & 540 & 547 & 537 \\
\hline$D_{19}$ & 529 & 516 & 551 & 540 & 492 & 480 & 470 & 459 & 465 & 454 \\
\hline$D_{20}$ & 429 & 425 & 440 & 431 & 375 & 364 & 295 & 284 & 250 & 243 \\
\hline$D_{21}$ & 265 & 282 & 295 & 287 & 253 & 244 & 211 & 203 & 189 & 181 \\
\hline \multicolumn{11}{|c|}{$a^{\prime \prime}$} \\
\hline$D_{22}$ & {$[967]^{\mathrm{f}}$} & 965 & 970 & 962 & 974 & 969 & 969 & 967 & 966 & 968 \\
\hline$D_{23}$ & 928 & 923 & 922 & 925 & 930 & 932 & 930 & 932 & 932 & 933 \\
\hline$D_{24}$ & 844 & 837 & 844 & 845 & 846 & 846 & 844 & 844 & 845 & 844 \\
\hline$D_{25}$ & 749 & 747 & 746 & 743 & 747 & 745 & 746 & 744 & 747 & 744 \\
\hline$D_{26}$ & 711 & 711 & 712 & 711 & 705 & 706 & 700 & 700 & 699 & 695 \\
\hline$D_{27}$ & 542 & 544 & 570 & 553 & 538 & 538 & 533 & 534 & 531 & 530 \\
\hline$D_{28}$ & 442 & 441 & 448 & 447 & 440 & 431 & 435 & 429 & 434 & 432 \\
\hline$D_{29}$ & 315 & 258 & 330 & 283 & 263 & 256 & 256 & 248 & 250 & 242 \\
\hline$D_{30}$ & 191 & 171 & 198 & 187 & 174 & 155 & 159 & 140 & 143 & 126 \\
\hline
\end{tabular}

${ }^{a}$ Infrared spectroscopy of the liquid unless otherwise indicated, from Ref. [34].

${ }^{\mathrm{b}}$ B3LYP/aug-cc-pVTZ values scaled by 0.97 - this work (see text). The conformer used for the halophenols was "cis" with the $\mathrm{OH}$ in the phenyl plane and pointing towards the halogen. The conformer used for $o$-cresol was eclipsed, with one $\mathrm{C}-\mathrm{H}$ group of the methyl in the plane of the phenyl ring, and trans with this $\mathrm{C}-\mathrm{H}$ pointing away from the $\mathrm{OH}$ group, which is also in the plane of the phenyl ring and pointing away from the methyl group. We have noted the symmetric and asymmetric stretch, or the localized nature, of $D_{10}$ and $D_{12}$ explicitly.

${ }^{\mathrm{c}}$ Ordering of these vibrations was uncertain in Ref. [34].

${ }^{\mathrm{d}}$ Raman studies of liquid [25].

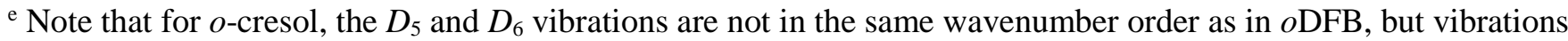
with similar motions have been given the same $D_{i}$ label; this is also the case for $D_{10}$ and $D_{11}$ for $o$-cresol and 
$o \mathrm{FPhOH}$, and for $D_{10}-D_{14}$ for $o \mathrm{ClPhOH}, o \mathrm{BrPhOH}$ and $o \mathrm{PhOH}$. The experimental wavenumbers have been ordered to match the calculated ordering.

${ }^{\mathrm{f}}$ Estimated value from Ref. [34]. 
Figure 1

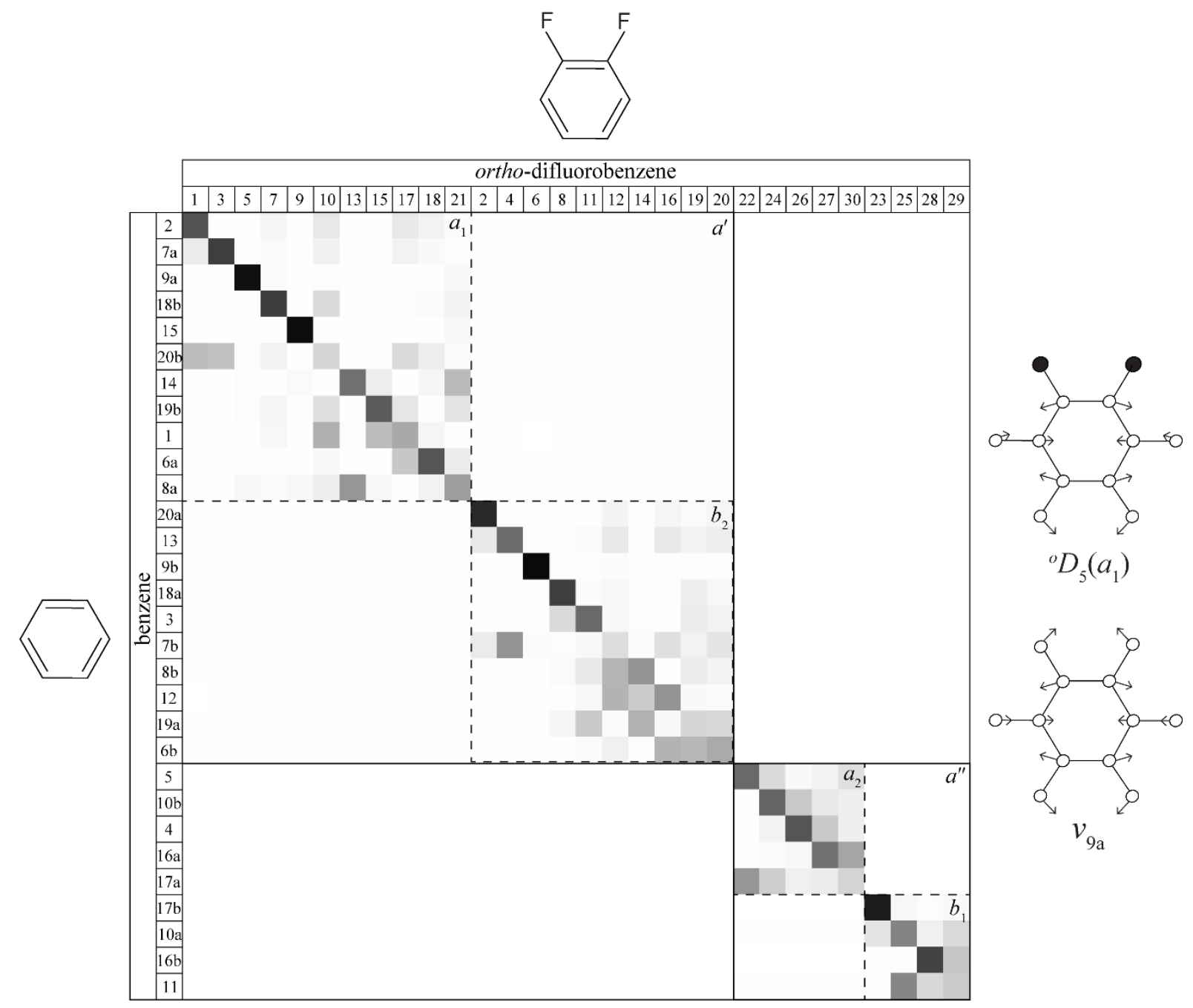


Figure 2

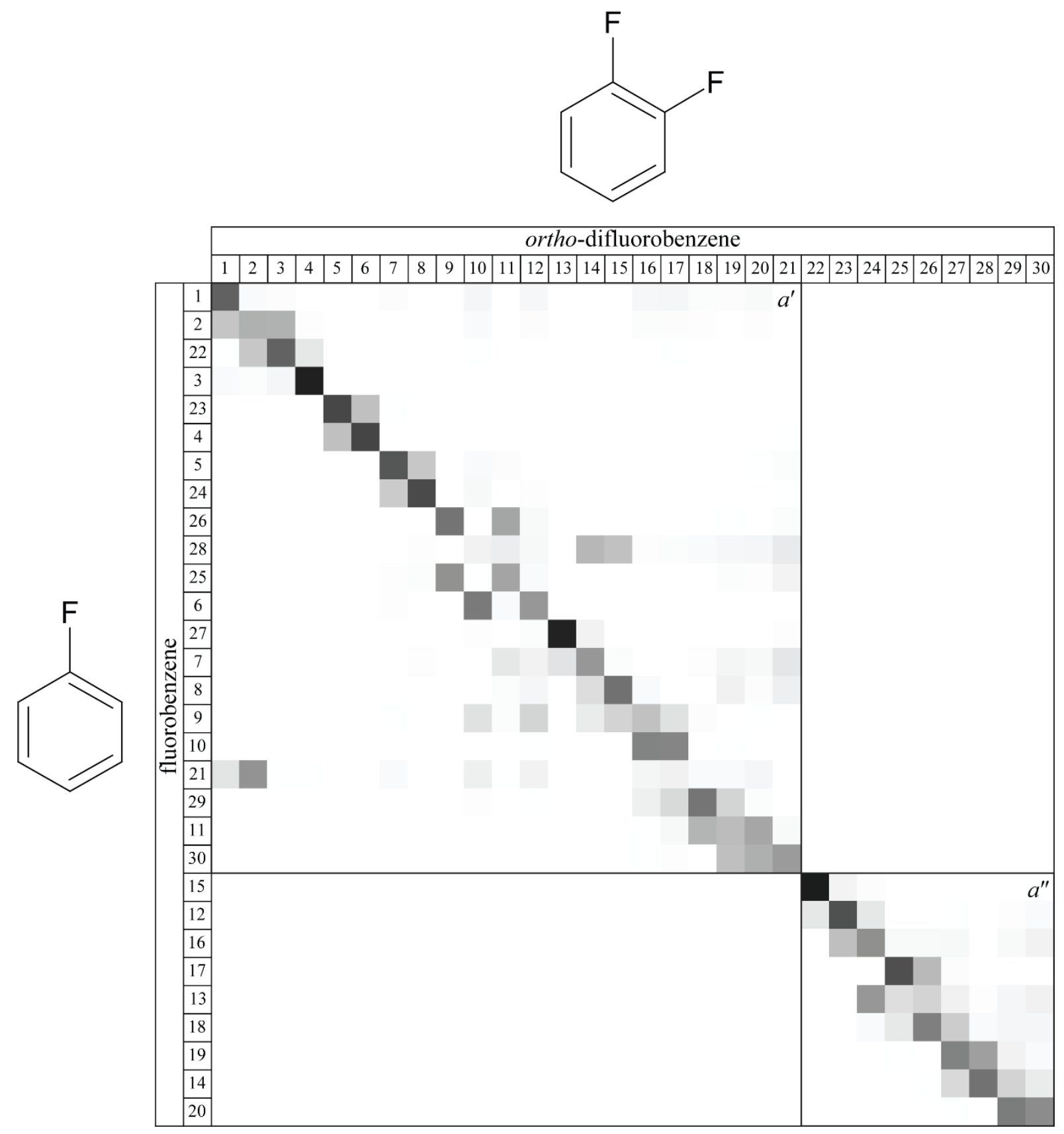


Figure 3
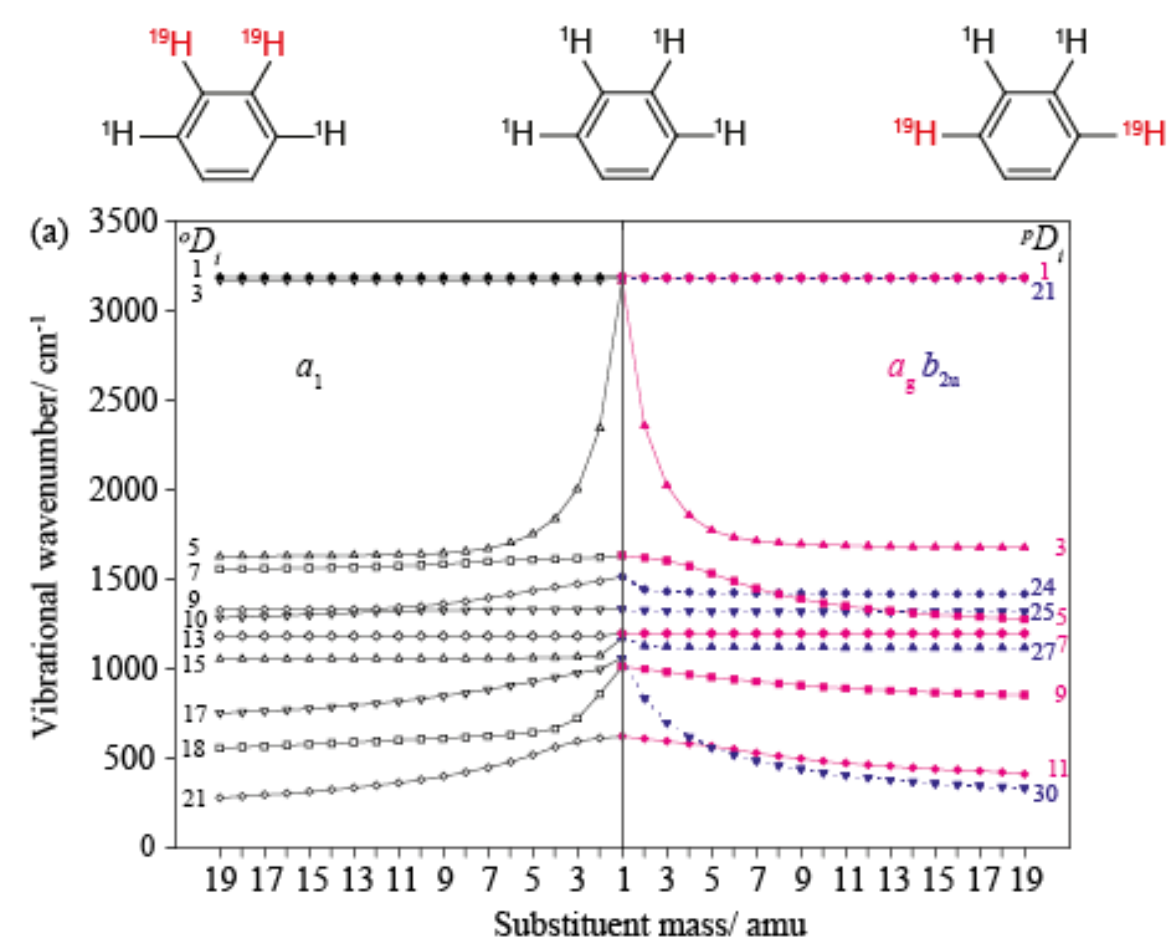
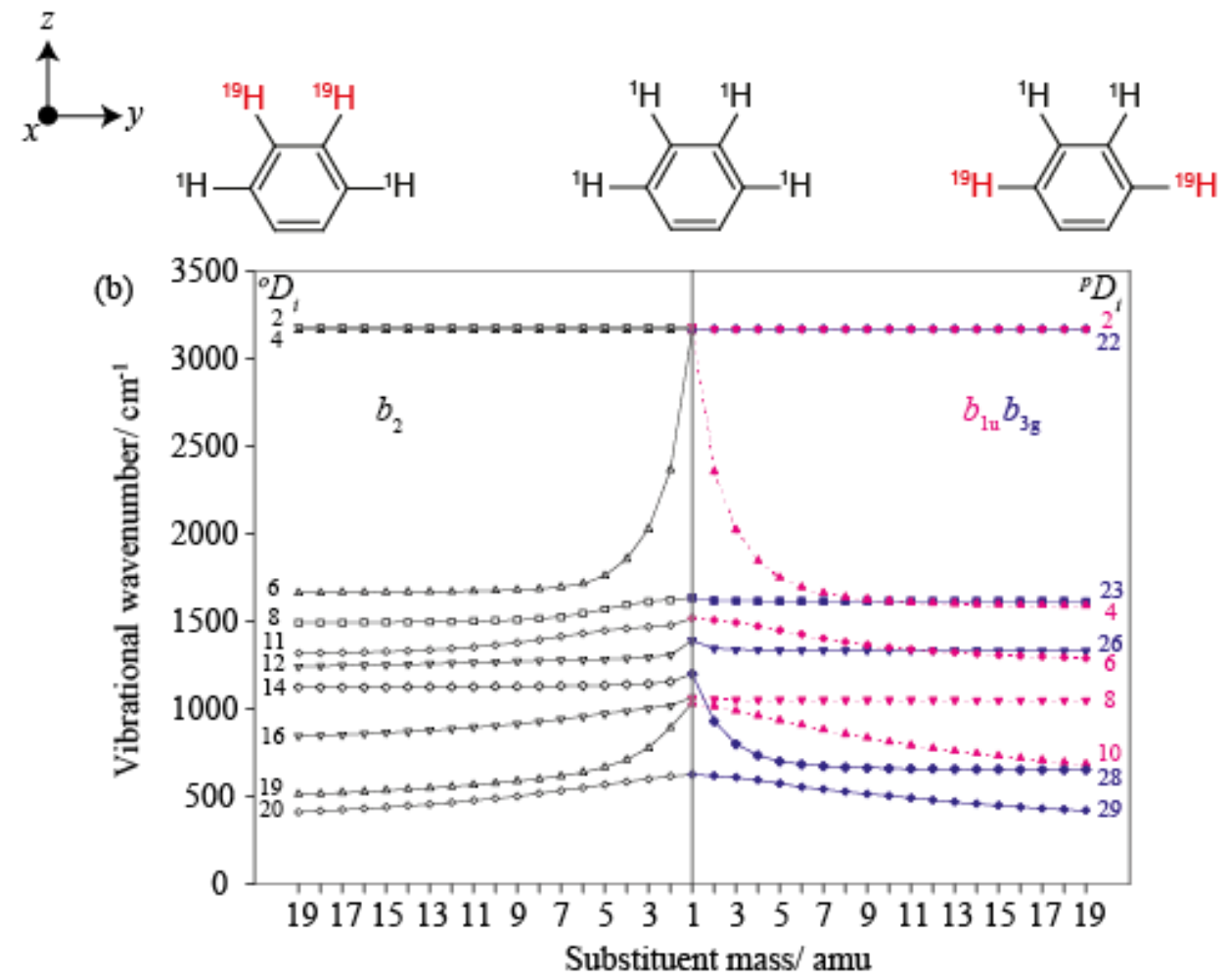


\section{Figure 4}
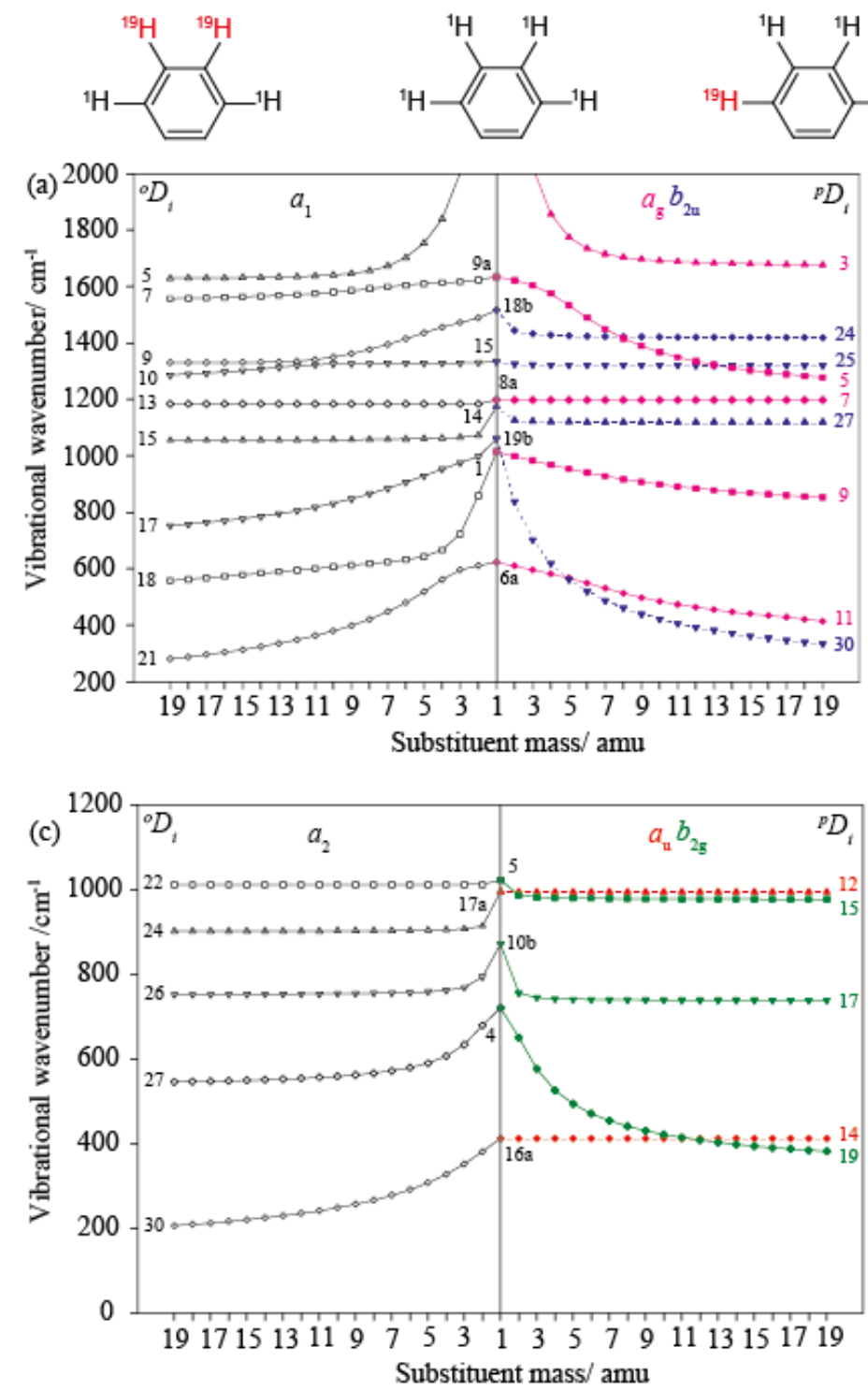
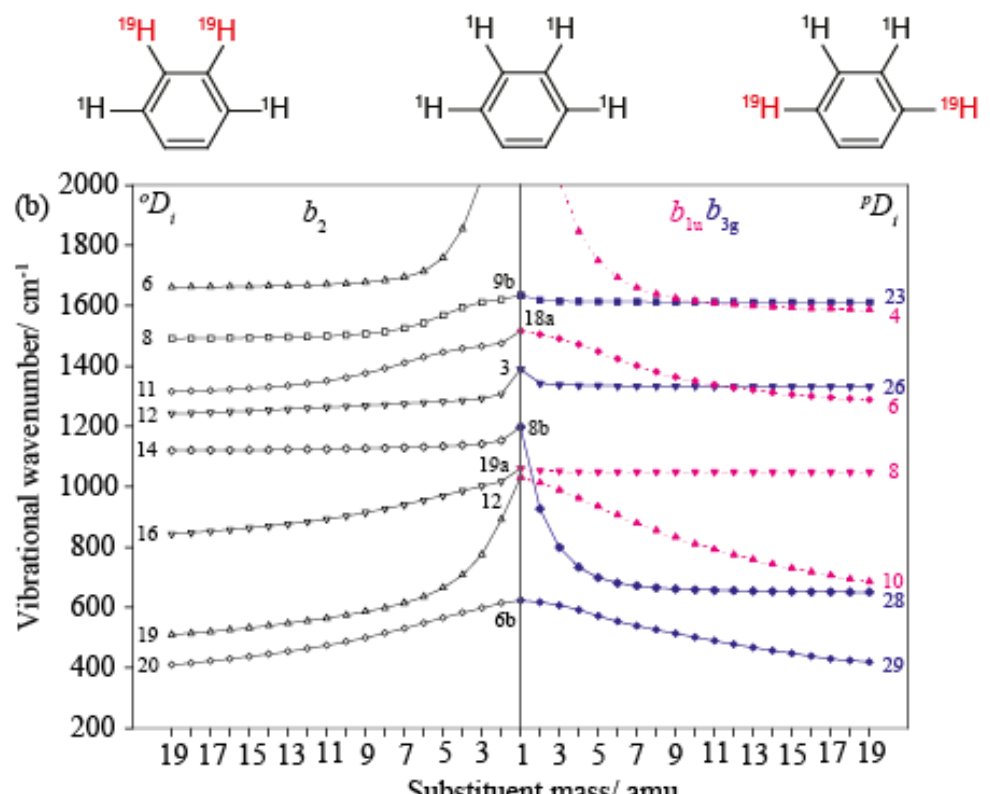

Substituent mass/amu

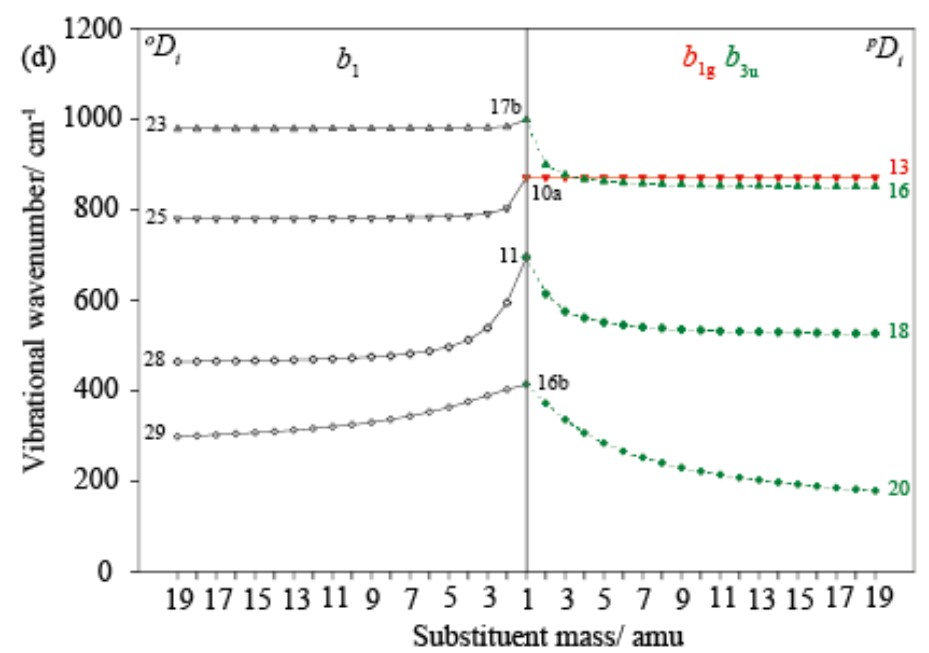


Figure 5

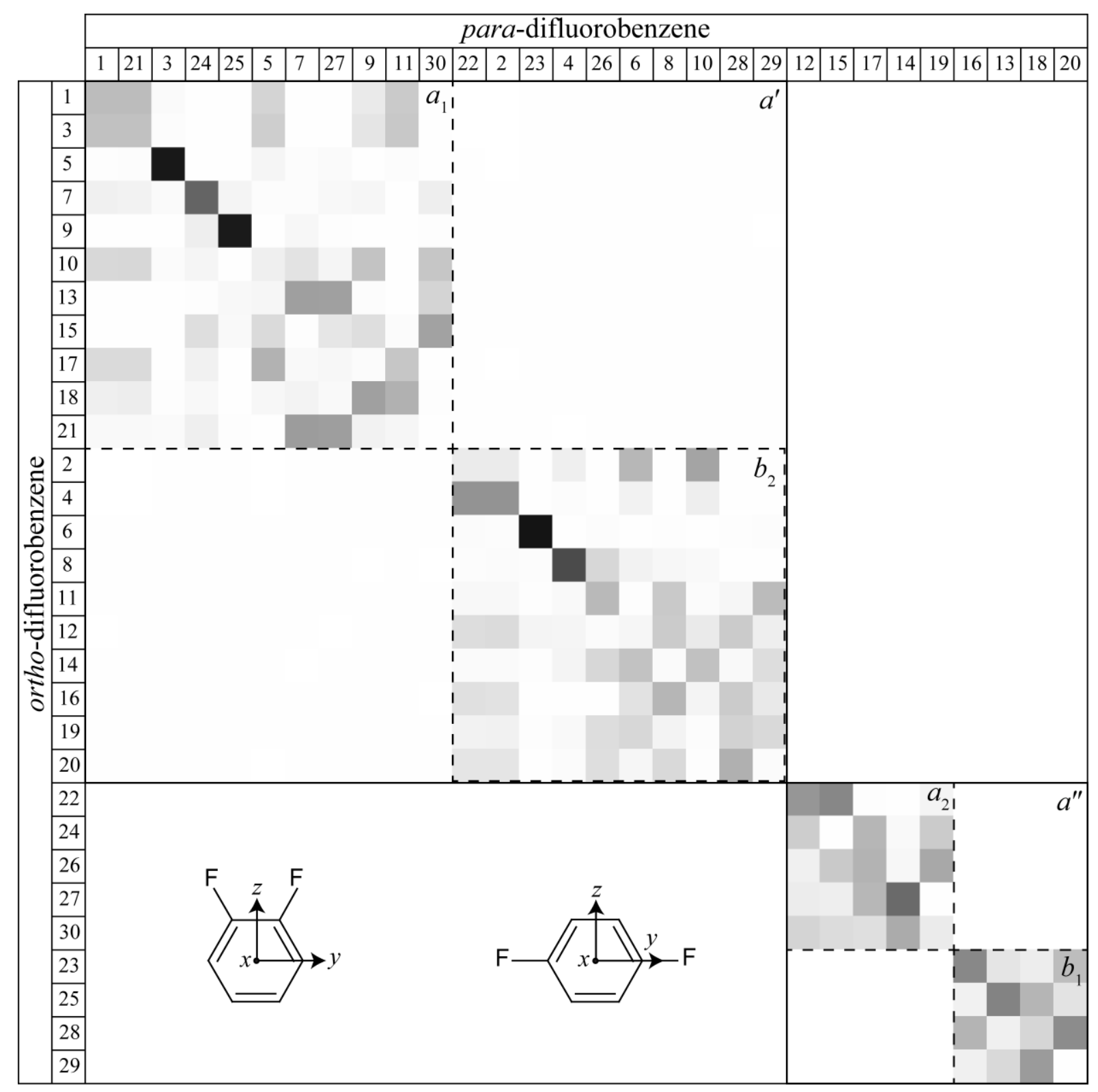




\section{Figure 6}

(a)

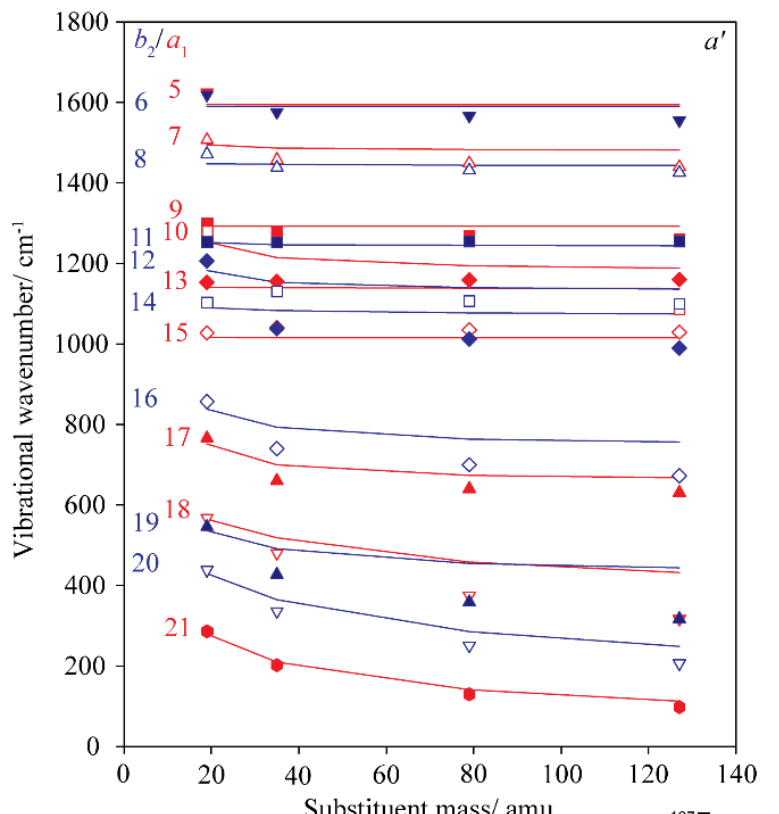

Substituent mass/amu (b)
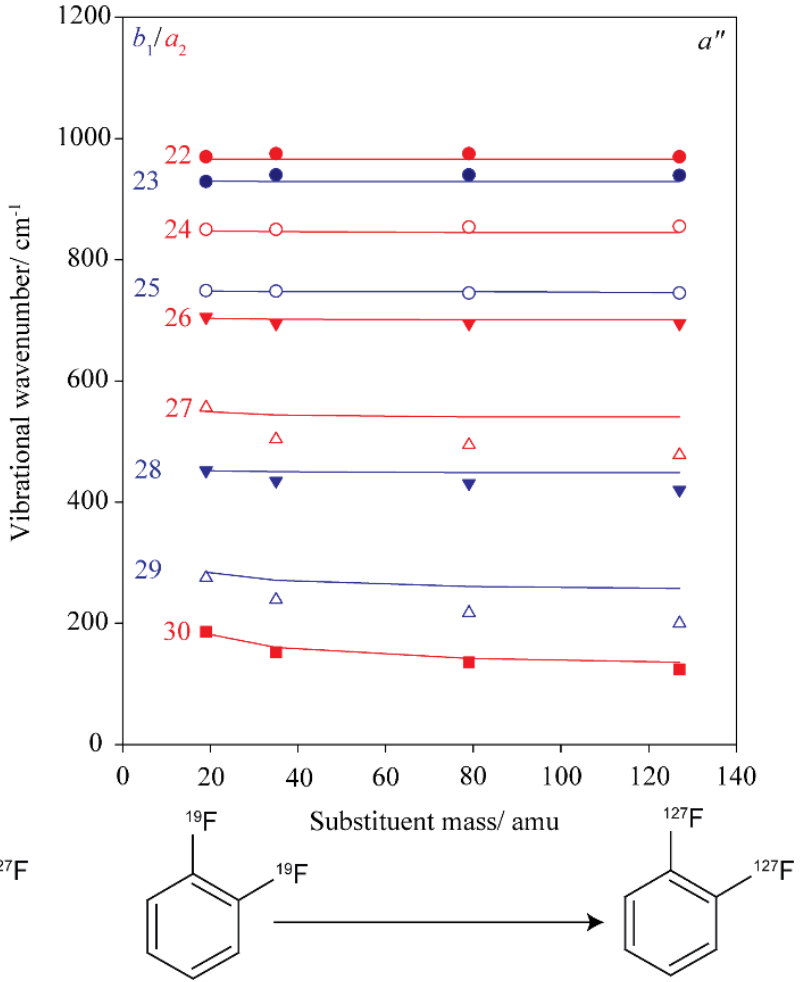
Figure 7

(a)

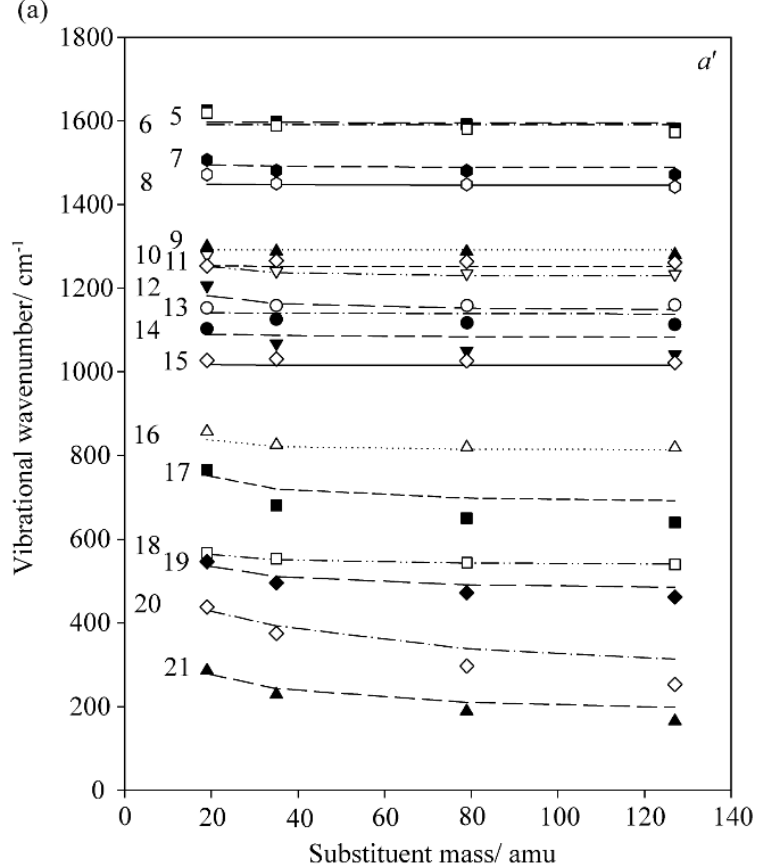

(b)

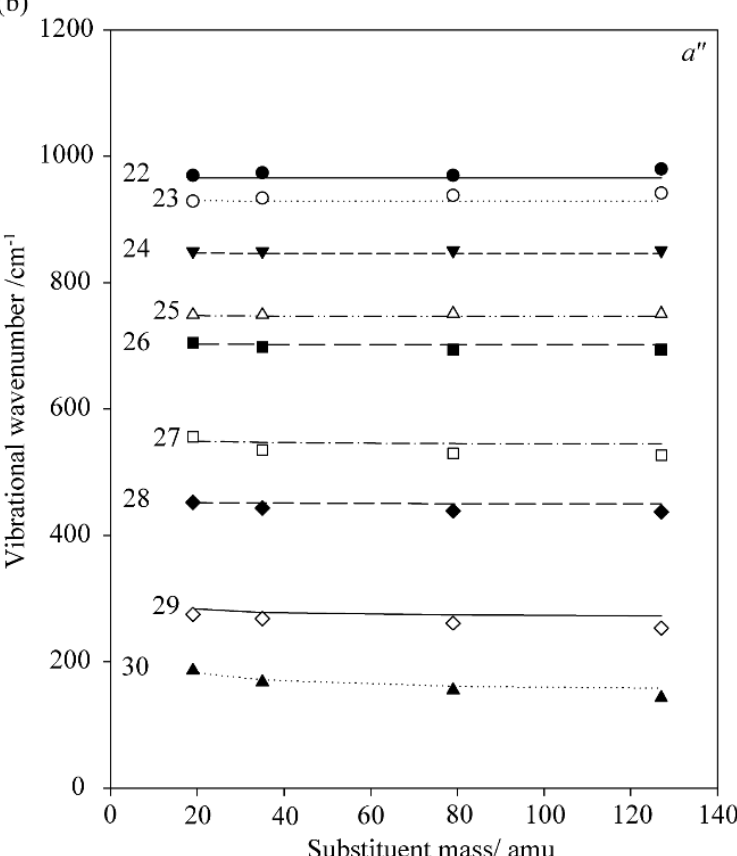

 
Figure 8

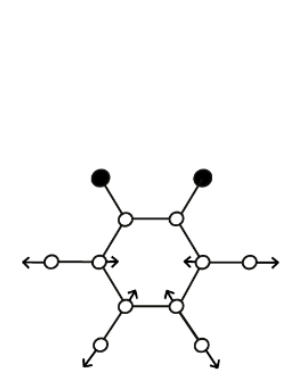

${ }^{o} D_{1}\left(a^{\prime}\right)$

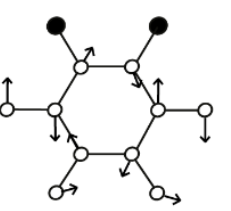

${ }^{o} D_{6}\left(a^{\prime}\right)$

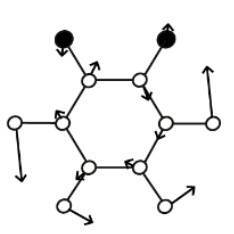

${ }^{o} D_{11}\left(a^{\prime}\right)$

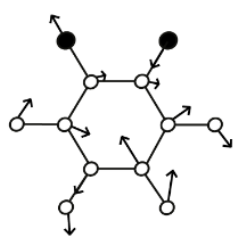

${ }^{o} D_{16}\left(a^{\prime}\right)$

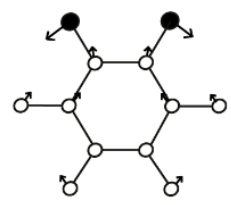

${ }^{o} D_{21}\left(a^{\prime}\right)$

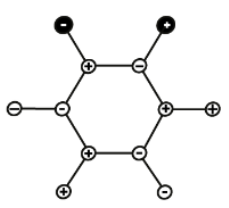

${ }^{o} D_{26}\left(a^{\prime \prime}\right)$

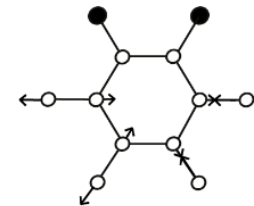

${ }^{o} D_{2}\left(a^{\prime}\right)$

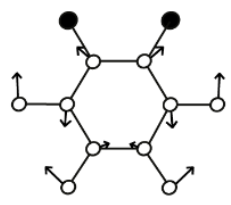

${ }^{o} D_{7}\left(a^{\prime}\right)$

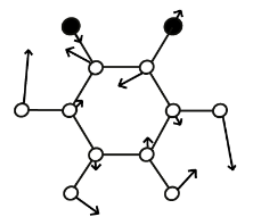

${ }^{o} D_{12}\left(a^{\prime}\right)$

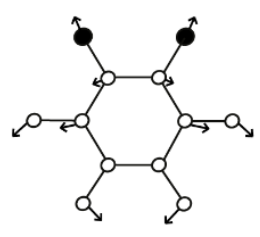

${ }^{o} D_{17}\left(a^{\prime}\right)$
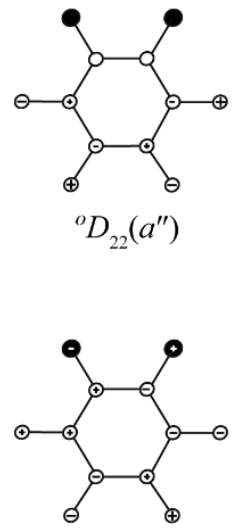

${ }^{o} D_{27}\left(a^{\prime \prime}\right)$

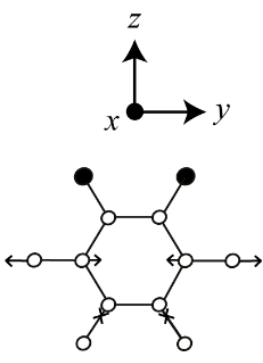

${ }^{o} D_{3}\left(a^{\prime}\right)$

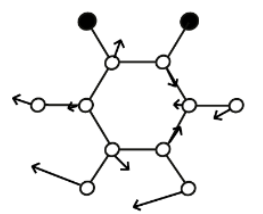

${ }^{o} D_{8}\left(a^{\prime}\right)$

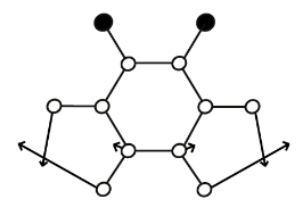

${ }^{o} D_{13}\left(a^{\prime}\right)$

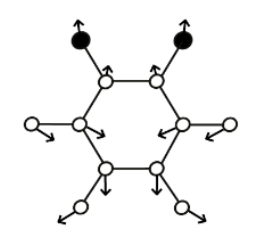

${ }^{o} D_{18}\left(a^{\prime}\right)$

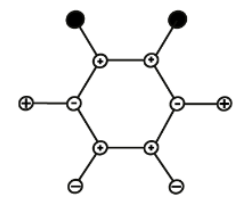

${ }^{o} D_{23}\left(a^{\prime \prime}\right)$

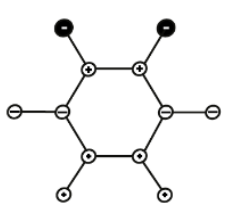

${ }^{o} D_{28}\left(a^{\prime \prime}\right)$

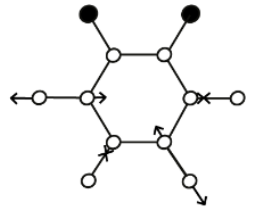

${ }^{o} D_{4}\left(a^{\prime}\right)$

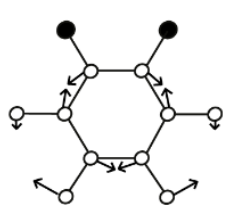

${ }^{o} D_{9}\left(a^{\prime}\right)$
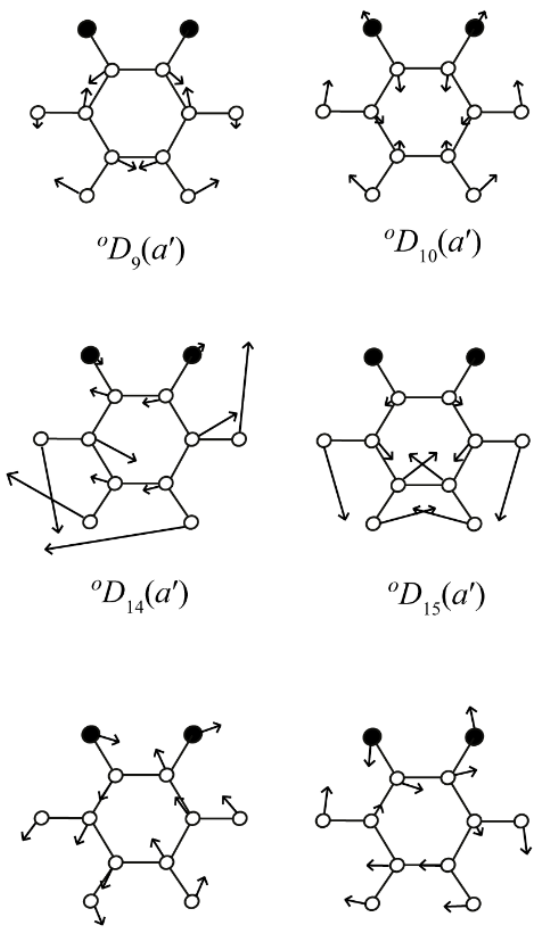

${ }^{o} D_{19}\left(a^{\prime}\right)$

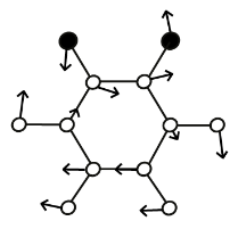

${ }^{o} D_{20}\left(a^{\prime}\right)$

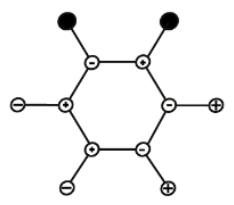

${ }^{o} D_{24}\left(a^{\prime \prime}\right)$
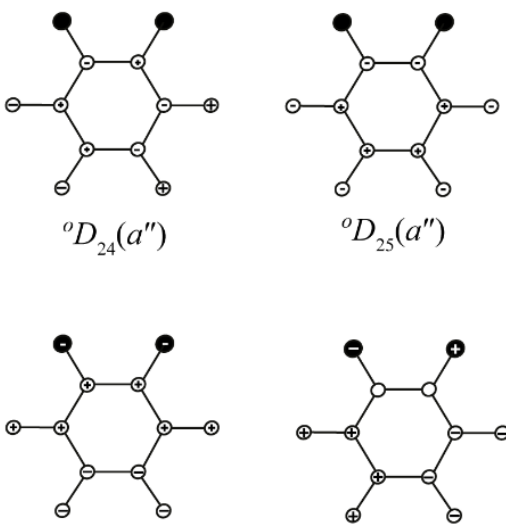

${ }^{o} D_{29}\left(a^{\prime \prime}\right)$

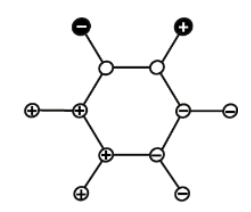

${ }^{o} D_{30}\left(a^{\prime \prime}\right)$ 
Figure 9

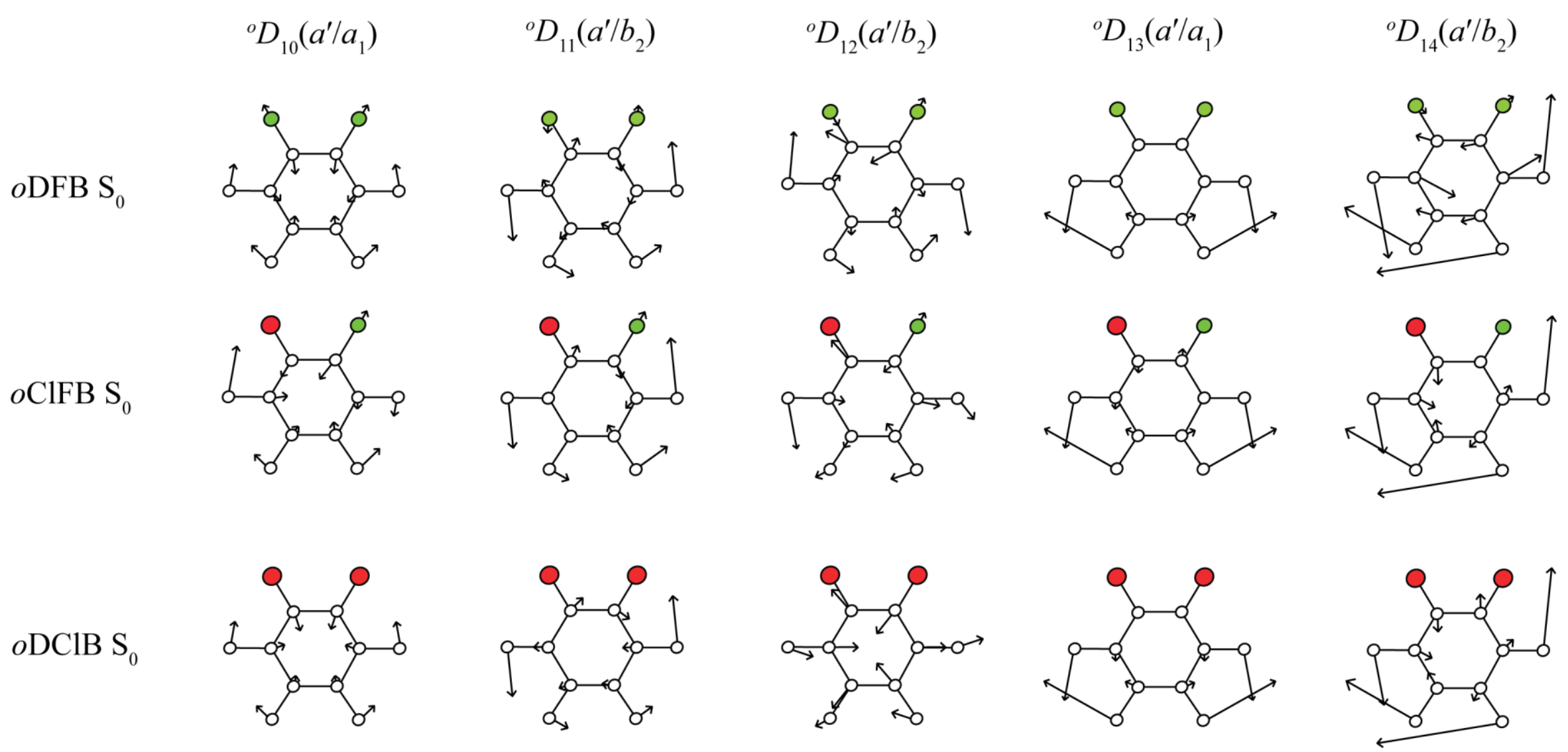




\section{References}

${ }^{1}$ E. B. Wilson, Jr, Phys. Rev. 45 (1934) 706.

${ }^{2}$ A. M. Gardner and T. G. Wright. J. Chem. Phys. 135 (2011) 114305.

${ }^{3} \mathrm{G}$. Varsányi, Assignments of the Vibrational Spectra of Seven Hundred Benzene Derivatives (Wiley, New York, 1974).

${ }^{4}$ G. Herzberg, Molecular Spectra and Molecular Structure II: Infrared and Raman Spectra of Polyatomic Molecules (Krieger, Malabar, 1991), p.272.

${ }^{5}$ R. S. Mulliken, J. Chem. Phys. 23 (1955) 1997.

${ }^{6}$ J. P. Harris, A. Andrejeva, W. D. Tuttle, I. Pugliesi, C. Schriever, and T. G. Wright, J. Chem. Phys. 141 (2014) 244315.

7 A. Andrejeva, W. D. Tuttle, J. P. Harris, and T. G. Wright, J. Chem. Phys. 143 (2015) 104312. ${ }^{8}$ A. Andrejeva, W. D. Tuttle, J. P. Harris, and T. G. Wright, J. Chem. Phys. 143 (2015) 244320.

${ }^{9}$ A. M. Gardner, A. M. Green, V M. Tamé-Reyes, V. H. K. Wilton and T. G. Wright J. Chem. Phys. 138 (2013) 134303.

${ }^{10}$ A. M. Gardner, A. M. Green, V. M. Tamé-Reyes, K. L. Reid, J. A. Davies, V. H. K. Parkes and T. G. Wright J. Chem. Phys. 140 (2014) 114038.

${ }^{11}$ A. Andrejeva, A. M. Gardner, W. D. Tuttle, and T. G. Wright, J. Molec. Spectrosc. 321 (2016) 28.

${ }^{12}$ A. M Gardner, W. D. Tuttle, L. Whalley, A. Claydon, J. H. Carter, and T G. Wright, J. Chem. Phys. 145 (2016) 124307.

${ }^{13}$ W. D. Tuttle, A. M. Gardner, L. E. Whalley and T. G. Wright, J. Chem. Phys. 146 (2017) 244310.

${ }^{14}$ W. D. Tuttle, A. M Gardner, K. B. O’Regan, W. Malewicz, and T. G. Wright, J. Chem. Phys. 146 (2017) 124309.

${ }^{15}$ A. M. Gardner, W. D. Tuttle, P. Groner, and T. G. Wright, J. Chem. Phys. 146 (2017) 124308.

${ }^{16}$ W. D. Tuttle, A. M. Gardner, and T. G. Wright, Chem. Phys. Lett. 684 (2017) 339.

${ }^{17}$ W. D. Tuttle, A. M. Gardner, A. Andrejeva, F. M. S. Jones, J. C. A. Wakefield and T. G. Wright, J. Molec. Spec. (unpublished).

${ }^{18}$ Gaussian 09, Revision E.01, M. J. Frisch, G. W. Trucks, H. B. Schlegel, G. E. Scuseria, M. A. Robb, J. R. Cheeseman, G. Scalmani, V. Barone, B. Mennucci, G. A. Petersson, H. Nakatsuji, M. Caricato, X. Li, H. P. Hratchian, A. F. Izmaylov, J. Bloino, G. Zheng, J. L. Sonnenberg, M. Hada, 
M. Ehara, K. Toyota, R. Fukuda, J. Hasegawa, M. Ishida, T. Nakajima, Y. Honda, O. Kitao, H. Nakai, T. Vreven, J. A. Montgomery, Jr., J. E. Peralta, F. Ogliaro, M. Bearpark, J. J. Heyd, E. Brothers, K. N. Kudin, V. N. Staroverov, R. Kobayashi, J. Normand, K. Raghavachari, A. Rendell, J. C. Burant, S. S. Iyengar, J. Tomasi, M. Cossi, N. Rega, J. M. Millam, M. Klene, J. E. Knox, J. B. Cross, V. Bakken, C. Adamo, J. Jaramillo, R. Gomperts, R. E. Stratmann, O. Yazyev, A. J. Austin, R. Cammi, C. Pomelli, J. W. Ochterski, R. L. Martin, K. Morokuma, V. G. Zakrzewski, G. A. Voth, P. Salvador, J. J. Dannenberg, S. Dapprich, A. D. Daniels, Ö. Farkas, J. B. Foresman, J. V. Ortiz, J. Cioslowski, and D. J. Fox, Gaussian, Inc., Wallingford CT, 2009.

19 I. Pugliesi and K. Muller-Dethlefs, J. Phys. Chem. A 110 (2006) 4657. A free download of the software can be found at http://www.fclab2.net

20 J. H. S. Green, Spectrochim. Acta. 25A (1970) 1913.

${ }^{21}$ J. H. S. Green, W. Kynaston and H. M. Paisley, J. Chem. Soc. 473 (1963).

22 D. Steele, Spectrochim. Acta 18 (1962) 915.

${ }^{23}$ G. Nonnenmacker and R. Mecke, Spectrochim. Acta 17 (1961) 1049.

${ }^{24}$ M. Brigodiot and J.-M. Lebas, J. Chim. Phys. 62 (1965) 347.

${ }^{25}$ E. Herz, Monatsch. 76 (1946) 1.

${ }^{26}$ A. K. Swinn and S. H. Kable, J. Molec. Spectrosc. 191 (1998) 49.

27 S. A. Kudchadker, A. P. Kudchadker, R. C. Wilhoit and B. J. Zwolinski, Thermochim. Acta 30 (1979) 319.

${ }^{28}$ S. J. Greaves and W. P. Griffith, Spectrochim. Acta 47A (1991) 133.

${ }^{29}$ H. W. Wilson, Spectrochim. Acta 30A (1974) 2141.

${ }^{30}$ A. Hidalgo and C. Otero, Spectrochim. Acta 16 (1960) 528.

${ }^{31}$ T. Y. Koh, S. J. Greaves and W. P. Griffith, Spectrochim. Acta 50A (1994) 857.

32 S. L. N. G. Krishnamuchari, Curr. Sci. 25 (1956) 185.

${ }^{33}$ N. A. Narasimhan and J. R. Nielsen, J. Chem. Phys. 24 (1956) 433.

${ }^{34}$ J. H. S. Green, Spectrochim. Acta 27A (1971) 2199. 